$1-15-2022$

\title{
Novel Oncogenic Transcription Factor Cooperation in RB-Deficient Cancer
}

Amy C. Mandigo

Ayesha A Shafi

Jennifer J McCann

Wei Yuan

Talya Laufer

See next page for additional authors

Follow this and additional works at: https://jdc.jefferson.edu/cbfp

Part of the Medical Cell Biology Commons, and the Oncology Commons Let us know how access to this document benefits you

This Article is brought to you for free and open access by the Jefferson Digital Commons. The Jefferson Digital Commons is a service of Thomas Jefferson University's Center for Teaching and Learning (CTL). The Commons is a showcase for Jefferson books and journals, peer-reviewed scholarly publications, unique historical collections from the University archives, and teaching tools. The Jefferson Digital Commons allows researchers and interested readers anywhere in the world to learn about and keep up to date with Jefferson scholarship. This article has been accepted for inclusion in Department of Cancer Biology Faculty Papers by an authorized administrator of the Jefferson Digital Commons. For more information, please contact: JeffersonDigitalCommons@jefferson.edu. 


\section{Authors}

Amy C. Mandigo, Ayesha A Shafi, Jennifer J McCann, Wei Yuan, Talya Laufer, Denisa Bogdan, Lewis Gallagher, Emanuela Dylgjeri, Galina Semenova, Irina A Vasilevskaya, M J Schiewer, Chris M McNair, Johann S de Bono, and Karen E Knudsen 


\section{Novel Oncogenic Transcription Factor Cooperation in RB-Deficient Cancer}

Amy C. Mandigo', Ayesha A. Shafi', Jennifer J. McCann', Wei Yuan², Talya S. Laufer', Denisa Bogdan², Lewis Gallagher', Emanuela Dylgjeri', Galina Semenova', Irina A. Vasilevskaya', Matthew J. Schiewer, ${ }^{1,3,4}$, Chris M. McNair ${ }^{4}$, Johann S. de Bono ${ }^{2}$, and Karen E. Knudsen ${ }^{1,3,4,5,6}$

\section{ABSTRACT}

The retinoblastoma tumor suppressor $(\mathrm{RB})$ is a critical regulator of E2F-dependent transcription, controlling a multitude of protumorigenic networks including but not limited to cell-cycle control. Here, genome-wide assessment of E2F1 function after RB loss in isogenic models of prostate cancer revealed unexpected repositioning and cooperation with oncogenic transcription factors, including the major driver of disease progression, the androgen receptor (AR). Further investigation revealed that observed AR/E2F1 cooperation elicited novel transcriptional networks that promote cancer phenotypes, especially as related

\section{Introduction}

The retinoblastoma tumor suppressor (RB) has a well-described role in tumor development and progression by serving as a transcriptional modulator. Canonically, RB functions through binding to E2F transcription factors, preventing the transcription of key genes responsible for cell-cycle progression. In response to mitogenic stimuli, RB becomes inactivated through a series of phosphorylation events in which cyclin-dependent kinase (CDK) and cyclin complexes hyperphosphorylate $\mathrm{RB}$, resulting in a conformational change and release from $\mathrm{E} 2 \mathrm{~F}$ factors $(1,2)$. As RB functions as a negative regulator of $\mathrm{E} 2 \mathrm{~F}$-dependent transcription, it is unsurprising that deep deletion of the RB1 gene is common in human malignancies (3-11). Further, loss of RB has been utilized as a prognostic marker of advanced disease and predictor of poor patient outcome (12-16). Although RB is considered a master regulator of cell-cycle control, recent studies revealed that loss of

'Department of Cancer Biology, Sidney Kimmel Cancer Center, Thomas Jefferson University, Philadelphia, Pennsylvania. ${ }^{2}$ The Institute of Cancer Research, London, UK; The Royal Marsden NHS Foundation Trust, London, UK. ${ }^{3}$ Department of Urology, Sidney Kimmel Cancer Center, Thomas Jefferson University, Philadelphia, Pennsylvania. ${ }^{4}$ Sidney Kimmel Cancer Center, Thomas Jefferson University, Philadelphia, Pennsylvania. ${ }^{5}$ Department of Medical Oncology, Sidney Kimmel Cancer Center, Thomas Jefferson University, Philadelphia, Pennsylvania. ${ }^{6}$ Department of Radiation Oncology, Sidney Kimmel Cancer Center, Thomas Jefferson University, Philadelphia, Pennsylvania.

Note: Supplementary data for this article are available at Cancer Research Online (http://cancerres.aacrjournals.org/).

Corresponding Author: Karen E. Knudsen, Thomas Jefferson University, 233 South 10th Street, BLSB 1050, Philadelphia, PA 19107. Phone: 215-503-5692; E-mail: karen.knudsen@jefferson.edu

Cancer Res 2022;82:221-34

doi: 10.1158/0008-5472.CAN-21-1159

This open access article is distributed under Creative Commons AttributionNonCommercial-NoDerivatives License 4.0 International (CC BY-NC-ND).

(C)2021 The Authors; Published by the American Association for Cancer Research to evasion of cell death. These observations were reflected in assessment of human disease, indicating the clinical relevance of the AR/E2F1 cooperome in prostate cancer. Together, these studies reveal new mechanisms by which RB loss induces cancer progression and highlight the importance of understanding the targets of E2F1 function.

Significance: This study identifies that RB loss in prostate cancer drives cooperation between $\mathrm{AR}$ and $\mathrm{E} 2 \mathrm{~F} 1$ as coregulators of transcription, which is linked to the progression of advanced disease.

RB in lung and prostate cancers does not correlate with increased proliferation $(17,18)$, yet is tightly associated with aggressive disease and poor outcome, confirming the wealth of preclinical evidence showing that RB plays pleiotropic roles in cancer control well beyond regulation of the cell cycle. Recent functional analysis using genome-wide strategies and molecular assessment revealed that RB loss likely results in rewiring of a multitude of oncogenic transcription factors that play a role in cancer, including but not limited to E2F1.

E2F1 derepression is a hallmark of RB loss and promotes tumorigenic phenotypes. Investigation of the molecular consequence of RB loss on E2F1 function revealed an expanded E2F1 cistrome and gained E2F1 transcriptional control of a myriad of genes and pathways that promote cancer progression including the regulation of cancer metabolism $(18,19)$. Assessment of gained E2F1 function after RB depletion across a castration-resistant prostate cancer (CRPC) tumor data set revealed positive correlations between E2F1 and novel target gene expression exclusively in RB-depleted tumors, validating that this gained E2F1 function after RB depletion is likely clinically significant (19). RB loss and gained E2F1 function results in gene expression signatures that accurately predict $\mathrm{RB}$ depletion in human tumors and are associated with poor patient outcome $(16,18)$. Thus, changes in E2F1 function play a significant role in the clinical assessment of RB status, which can be utilized as a prognostic marker of disease stage, a predictor of patient outcome, and a biomarker for therapy response. Despite these significant advances, the mechanism by which E2F1 function serves to promote aggressive disease remains largely unknown. Notably, prior unbiased assessment of E2F1 repositioning after RB loss revealed enriched E2F1 binding in association with motifs of known oncogenic transcription factors including CCCTC-binding factor and forkhead box A1 (FOXA1; refs. 18, 19). Thus, it has been hypothesized that E2F1 genomic binding events not only expand in the context of RB loss, but also expand the repertoire of functions utilized by E2F1 to promote cancer phenotypes. Assessment of the E2F1 cistrome after RB loss revealed enrichment of several oncogenic transcription factors of cancer relevance, including those that are drivers of disease such as the androgen receptor (AR). 
The concept of E2F1 cooperation with oncogenic transcription factors was challenged herein, using prostate cancer as a model system. In prostate cancer, $\mathrm{RB}$ loss has the known ability to induce resistance to androgen-deprivation therapy through $\mathrm{E} 2 \mathrm{~F} 1$ regulation of $\mathrm{AR}$, resulting in poor patient outcome $(16,20)$. Further, a previous study revealed that AR does in fact cooperate with E2F1 to regulate the transcription of several genes in hormone-sensitive prostate cancer (21). These studies, along with the identification of AR-associated motifs proximal to E2F1 binding after RB loss $(18,19)$, suggest that AR may contribute to the regulation of the expanded $\mathrm{E} 2 \mathrm{~F} 1$ cistrome. In brief, the studies here reveal that $\mathrm{E} 2 \mathrm{~F} 1$ binding is repositioned after $\mathrm{RB}$ loss to cooperate with $\mathrm{AR}$ and drive resultant gene expression networks that induce protumorigenic phenotypes. Key discoveries through use of isogenic human cancer cell models identified significant upregulation of E2F1 and AR co-occupancy on DNA after RB loss, which was dependent on AR activation, and further described as either constitutive co-occupied binding (CCB) or gained cooccupied binding (GCB) after RB loss. Further, AR and E2F1 transcriptional coregulation provides a protective advantage from apoptosis-inducing agents after RB depletion, rendering cancer cells less sensitive to clinically utilized therapies. Gene networks coordinately regulated by AR and E2F1 were subsequently validated in human tissue, thus confirming the clinical relevance of these newly discovered pathways downstream of RB. These studies provide a new perspective of RB-depleted transcriptional control and reveal a shift in understanding of the gene expression signatures currently utilized as clinical markers of disease progression.

\section{Materials and Methods}

\section{Cell lines, cell culture, and reagents}

C4-2 cells were obtained from and authenticated by ATCC. LnCaP95 (LN95) cells were received as a generous gift from Dr. Jun Luo at John Hopkins University. C4-2-derived cell lines were maintained in Improved Minimum Essential Medium (IMEM; Thermo Fisher Scientific, 10024CV), supplemented with 5\% heat-inactivated fetal bovine serum (FBS), $1 \% \mathrm{~L}$-glutamine $(2 \mathrm{mmol} / \mathrm{L} / \mathrm{nmol} / \mathrm{L})$, and $1 \%$ penicillin-streptomycin (100 units/mL). LN95-derived cell lines were maintained in modified IMEM (Thermo Fisher Scientific, A1048801), supplemented with $10 \%$ CDT and $1 \%$ penicillin-streptomycin. All cells were maintained at $37^{\circ} \mathrm{C}$ with $5 \% \mathrm{CO}_{2}$. All cell lines were checked for Mycoplasma upon thawing.

\section{RB1 knockdown cell lines}

C4-2 isogenic models were previously developed as described (22). Briefly, cells were transfection with either an shRNA plasmid targeting RB (sequence: 5'-CGCATACTCCGGTTAGGACTGTTATGAA-3') or a control plasmid (MSCV donor; ref. 23). LN95 cells were transfected with either an shRNA plasmid targeting RB (sequence: $5^{\prime}$-GCAGTTCGATATCTACTGAAA-3') or a nonspecific control (sequence: $5^{\prime}$-GCTGAGGTGATAAACAGTTACA-3 ${ }^{\prime}$ ) as previously described (24). Newly generated LN95 RB-depleted (LN95-miCon and LN95-miRB) cell lines underwent three rounds of antibiotic selection with $2.5 \mu \mathrm{g} / \mathrm{mL}$ puromycin.

\section{Chromatin immunoprecipitation sequencing}

AR chromatin immunoprecipitation sequencing (ChIP-seq) was performed as previously described (18). Briefly, cells were plated in 5\% CDT media for 72 hours followed by three hours of $10 \mathrm{nmol} / \mathrm{L}$ DHT treatment. Cells were cross-linked with $1 \%$ fresh formaldehyde for 10 minutes at room temperature. Chromatin was sheared to approxi- mately 200 bp using a Diagenode Ultrasonicator for 30 cycles (30 seconds on, 30 seconds off). AR antibody utilized for ChIP-seq was purchased from MilliporeSigma (06-680). Validation by ChIP-qPCR was performed with AR antibody purchased from Abcam (ab74272). AR ChIP-seq libraries constructed using the Swift BioSciences ACCEL-NGS 2S Plus DNA Library kit with 10 ng of DNA. Sequencing was performed on NexTSeq 500 at the Jefferson University Sidney Kimmel Cancer Sequencing Core Facility. FASTQ files were assessed for quality using FASTQC v0.11.5. Reads were aligned to the human genome reference version hg19 using bowtie2 v2.3.271 with default parameters. Peak calling was performed using MACS2 v2.1.172 with combined replicates, utilizing a $q<0.05$ cutoff. Peak annotation and motif analysis performed using Homer v4.10.374 using the parameters indicated. Cis-regulatory element analysis was performed using CEAS v1.0.2. Motif analyses were performed through Homer v4.8.3 (25). AR ChIP-seq data have been deposited in the GEO repository with accession number GSE171511.

\section{Gene expression}

Cells were plated at equal densities in hormone-deficient media for 72 hours followed by 16 hours of $10 \mathrm{nmol} / \mathrm{L}$ DHT treatment. RNA was isolated using TRIzol (Invitrogen). RT-PCR was performed using the SuperScript VILO cDNA Synthesis Kit (Thermo Fisher Scientific). ABI StepOne Real-Time PCR using PowerSybr (Fisher Scientific, 43-676-59) was utilized to perform quantitative PCR (qPCR) analyses. Primer sequences are as follows: TNFAIP8 (Fw: 5'-CTCAAGCTCTTCTACGGGA-3'; Rev: 5'-CTCAGAGGCTCTGTACTCAG-3'); FKBP5 (Fw: 5'-AAATGAGTCACAGGTCATCAG-3' ${ }^{\prime}$; Rev: $5^{\prime}$-CTGGAACAGCTAGCAATTCC- $3^{\prime}$ ); FOXN3 (Fw: 5'-CAAATGCACCTACTGGGTGC-3'; Rev: 5'-CCCAATACTCTGACTCCTCTC-3'); MCM7 (Fw: 5' -AGTATGGGAACCAGTTGGT-3'; Rev: 5'-ATTTACCACTTCCCTCTCCT-3'); ESRP1 (Fw: 5'-AATATTGCCAAGGGAGGTG-3'; Rev: 5'-ACTTACAAACCTAACCAGAGC-3'); SGK1 (Fw: 5'-GTTCAGTCCATCTTGAAGATCTC-3'; Rev: 5'-AGAAGGACTTGGTGGAGGA-3'); $18 S$ (Fw: 5'-CGGCGACGACCCATTCGAAC-3'; Rev: 5'-GAATCGAACCCTGATTCCCCGTC-3' ${ }^{\prime}$.

\section{Immunoblotting}

C4-2-derived cells were plated in equal densities 5\% CDT media for 72 hours followed by 24 hours of treatment with $10 \mathrm{nmol} / \mathrm{L}$ DHT or vehicle. LN95-derived cells were plated in equal densities in $10 \% \mathrm{CDT}$ media for 24 hours and treated with $10 \mathrm{nmol} / \mathrm{L}$ DHT for 24 hours. To induce apoptosis, cells were treated with either $8 \mu \mathrm{mol} / \mathrm{L}$ cisplatin (Mylan Pharmaceuticals) or $10 \mathrm{ng} / \mathrm{mL}$ TNF $\alpha$ (R\&D Systems; 210-TA-005) in combination with $5 \mu \mathrm{g} / \mathrm{mL}$ of actinomycin D (Sigma-Aldrich; A1410) for 24 hours. Cell lysates were generated as previously described (26). Lysate was resolved by SDS-PAGE and transferred to polyvinylidene membrane. Proteins were analyzed using the following antibodies at 1:1,000 dilution-RB (BD Pharmingen 554136), AR (N-20, directed against amino acids $1-20$ by Bethyl Laboratories), E2F1 (Cell Signaling Technologies; 3742S), TNFAIP8 (Abcam; ab195810), PARP (Cell Signaling; 9542S), Caspase-3 (Cell Signaling; 9662S), cleaved caspase-3 (Cell Signaling; 9661S), FKBP5 (Thermo Fisher Scientific; PA1020), GAPDH (Santa Cruz Biotechnology; sc-25778), and Vinculin (Sigma-Aldrich; V9264).

\section{ChIP-qPCR}

qPCR analyses were performed on the ABI StepOne Real-Time PCR using PowerUPsybr (Fisher Scientific, A25742) primer sequences are 
listed below. For ChIP-qPCR, C4-2-shCon and C4-2-shRB cells were transfected as described below. Cells were moved 5\% CDT media following transfection for 72 hours and treated with $10 \mathrm{nmol} /$ L DHT for three hours. Primers are as follows: TNFAIP8 (Fw: 5'-GGGAGGGTAGGTAGCGTACT-3'; Rev: $5^{\prime}$ - CTACGGTTCTCAGCTTGGCA-3'); FKBP5 (Fw: 5'-TGTCCAGCCAGACCAAACAA-3'; Rev: 5'- TGTCCAGCCAGACCAAACAA-3'); CENPC (Fw: 5'-TCCATCACAAACTAGTATTGCC-3'; Rev: 5'-AGTACTCCAAAGGTTTCAAACG - $3^{\prime}$ ); FOXN3 (Fw: $5^{\prime}$-CAAATGCACCTACTGGGTG-3'; Rev: $5^{\prime}$-CCCAATACTCTGACTCCTCTC-3'); MCM7 (Fw: 5'-CTGGGAACCTTCCAACCAGG-3'; Rev: 5'-CAGTTCCCGTTTGACAAGCC- $3^{\prime}$ ); ESRP1 (Fw: $5^{\prime}$-GTCGTGGTTTGAAGGAGCCA-3'; Rev: $5^{\prime}$-GGATGTGGCTTTACCTGCCC-3'); FZD1 (Fw: 5'-TTCAAGGCTCCTCCCTCCTG-3'; Rev: 5'-TGTCAATCCCTCAACTCGCTC-3'); SGK1 (Fw: $5^{\prime}$-CTCTTCCCACCCACTTGTGC-3'; Rev: $5^{\prime}$-TTGAAAGGTGCCAGAGGAGAC-3'); Desert (Fw: 5'-CTAGGGTGGAGGTAGGG-3'; Rev: 5'-GCCCCAAACAGGAGTAATGA-3').

\section{RNA interference}

C4-2-shCon and C4-2-shRB cell lines were seeded on poly-Llysine-coated plates in 5\% FBS media for 24 hours. Cells were transfected with either scramble control (C4-2-shCon siCtl, C42-shRB siCtl), E2F1 (C4-2-shCon siE2F1, C4-2-shRB siE2F1), or AR (C4-2-shCon siAR, C4-2-shRB siAR) siRNA pools (Dharmacon) for eight hours according to the manufacturer's protocol. Following transfection, the cells were moved 5\% CDT media for 72 hours followed by appropriate $10 \mathrm{nmol} / \mathrm{L}$ DHT treatment.

\section{MTT cell viability assay}

C4-2 and LN95 isogenic pairs were seeded at equal densities in a 96-well plate. C4-2-derived models were seeded in 5\% CDT for 72 hours followed by 24 hours of $10 \mathrm{nmol} / \mathrm{L}$ DHT. LN95-derived models were seeded in $10 \%$ CDT for 48 hours. Cells were treated with varying concentrations of TNF $\alpha(0.1$ to $50 \mathrm{ng} / \mu \mathrm{L})$ and $5 \mu \mathrm{g} / \mathrm{mL}$ of actinomycin D for 24 hours. Cells were treated following manufacturer's instructions with kit reagents (Sigma-Aldrich 11465007001), and absorbance was measured at $625 \mathrm{~nm}$ on a BioTek Synergy HT microplate reader and analysis using BioTek Gen5 2.09 software.

\section{Royal Marsden Hospital cohort}

Royal Marsden Hospital (RMH) cohort patient samples $(n=98)$ were collected and analyzed with written informed consent under the CCR2472 protocol approved by the Ethics committee at the Royal Marsden NHS Foundation Trust Hospital (London, UK).

\section{RMH sample RNA-seq}

RNA, from fresh tissue biopsy, quality was analyzed using Agilent RNA ScreenTape assay (Agilent). Total RNA (500 ng) was used for library preparation using the NEBNext rRNA depletion kit followed by NEBNext Ultra II directional RNA assay kit as per manufacturers protocol (New England Biolabs). Library quality was confirmed using the Agilent High sensitivity D1000 ScreenTape Assay (Agilent). The libraries were quantified and normalized by qPCR using the Generead Library Quant Kit (Qiagen). Library clustering and sequencing were performed on the Illumina NovaSeq 6000. The libraries were run across two lanes of an Illumina NovaSeq S2 flowcell using 150 base pair (bp) pair-end v1 Kit and eight base pair dual indexes. Base-calling and quality scoring were performed using Real-Time Analysis (version v3.4.4) and FASTQ file generation and demultiplexing using Illumina bcl2fastq2 (version 2.20). CRPC transcriptomes reads were aligned to the human reference genome (GRCh37/hg19) using TopHat2 (version 2.0.7). Gene expression, fragments per kilobase of transcript per million mapped reads, was calculated using Cufflinks. AR activity score was an accumulation measurement of AR pathway activity based on 43 genes regulated by AR in prostate cancer cell lines and metastatic prostate cancer as previously described (27).

\section{Statistical analysis}

All experiments were performed in technical triplicate with at least three biological replicates per condition. Data are displayed as mean \pm standard error of the mean. Statistical significance $(P<0.05)$ was determined using Student $t$ test, one-way ANOVA, and two-way ANOVA on GraphPad Prism Software as appropriate.

\section{Results}

\section{RB depletion reprograms AR binding promoting increased AR/} E2F1 co-occupancy

The RB tumor suppressor regulates multiple transcriptional networks associated with antitumorigenic activity, including regulation of cell-cycle control, DNA repair, and metabolism $(1,2$, 19, 28-30). Conversely, RB depletion leads to network derepression and rewiring of the E2F1 cistrome, which has been implicated in tumor progression $(18,19)$. However, the mechanisms driving this gained E2F1 cistrome and downstream transcriptional regulation remain poorly understood. To examine the impact of AR on E2F1 binding after RB loss, $A R$ binding was examined in response to $\mathrm{RB}$ depletion via ChIP followed by DNA sequencing (ChIP-seq) after three hours of androgen stimulation. RB depletion resulted in a shift in AR binding with 8,044 sites lost and 5,037 new sites gained when compared with RB intact conditions (Fig. 1A). Peak annotation assessment revealed a preference for AR binding at intronic and distal intergenic regions in both RB intact and RB-depleted conditions (Fig. 1B), indicating that although there is a shift in AR binding, the preference for distal regions of DNA is not altered after $\mathrm{RB}$ depletion. To further assess the 5,037 binding sites that were gained after RB loss, known motif analysis was performed on sites that were exclusive to the RB-depleted condition. The motifs of known AR-associated proteins were enriched such as FOXA1, a pioneer factor for AR transcription that plays a significant role in chromatin remodeling providing access for AR to bind DNA and forkhead box M1 (FOXM1), a cofactor of AR that is highly expressed in prostate cancer and associated with disease progression (31-33). Enrichment of these known AR-associated motifs suggests that AR maintains canonical regulatory mechanisms at these gained binding sites. Interestingly, previously reported E2F1-associated motifs including nuclear factor-1 halfsite (NF1halfsite; ref. 18) were also enriched in known and de novo motif analysis of AR binding exclusively after RB loss (Fig. 1C; Supplementary Fig. S1), suggesting that AR may be localized to sites associated with E2F1 after RB depletion. In conclusion, these data reveal for the first time that RB depletion promotes a shift in $\mathrm{AR}$ binding to sites near E2F1-associated motifs, introducing the potential for cooperation between $\mathrm{AR}$ and $\mathrm{E} 2 \mathrm{~F} 1$ in late-stage, RB-deficient disease.

To further explore this concept of enhanced AR and E2F1 cooperation after RB loss, AR binding was directly compared with previously published E2F1 binding in the same models under identical conditions. Binding sites having an overlap of $1 \mathrm{bp}$ or more were designated as co-occupied (Fig. 1D, top). Assessment in control 

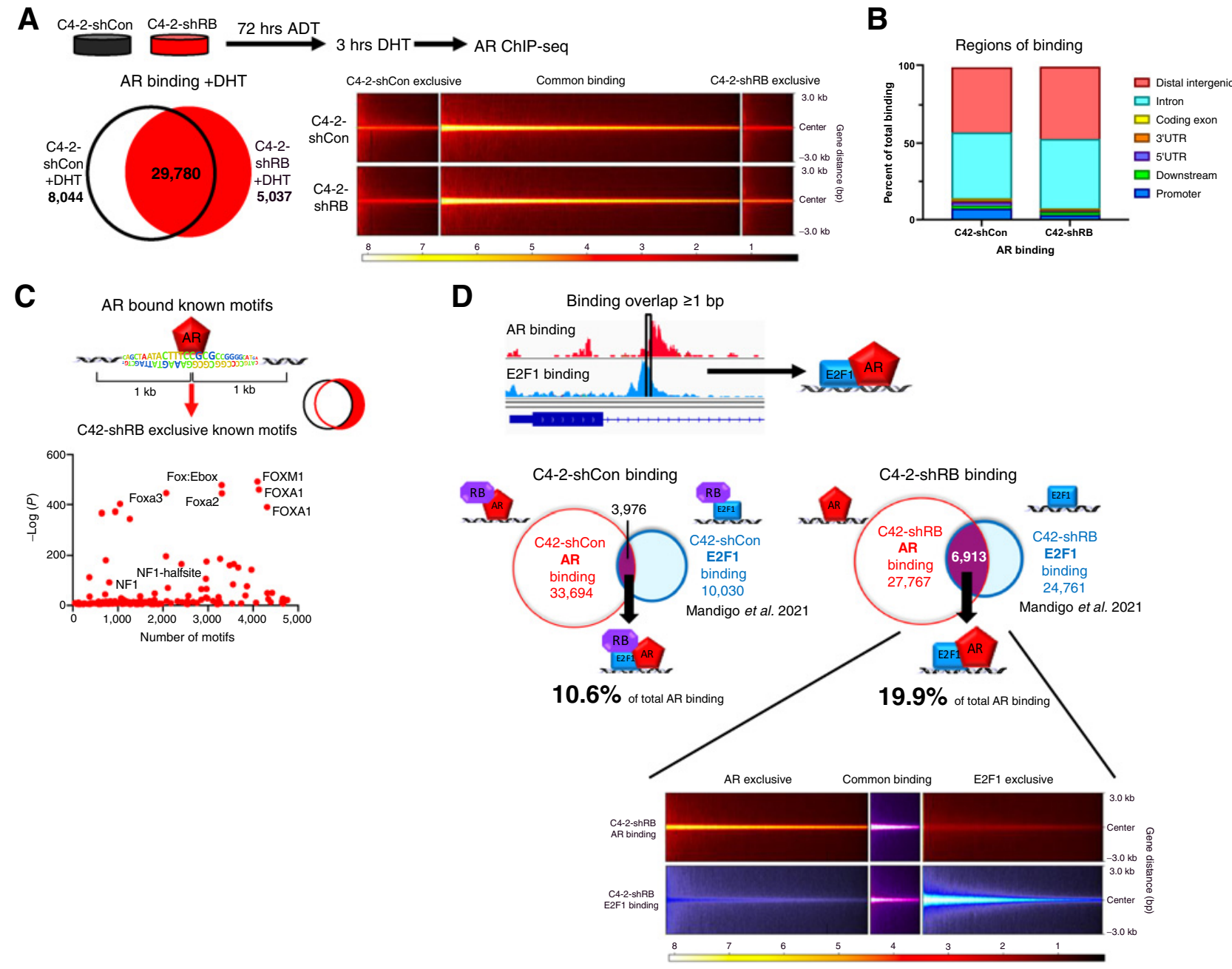

Figure 1.

RB depletion reprograms AR binding promoting increased AR and E2F1 co-occupancy. A, AR ChIP-seq performed in the C4-2-shCon and C4-2-shRB models after 72 hours of androgen depletion and 3 hours of DHT stimulation. Number of binding sites in each condition is shown via Venn diagram (left), along with binding intensity (right). B, Regions of AR binding for each model were determined using the cis-regulatory element annotation system (CEAS) package. Binding regions are represented as a percentage of total binding. $\mathbf{C}$, Known motif analysis within a $1 \mathrm{~kb}$ window from the center of AR binding exclusively after RB1 depletion in the C4-2-shRB model. Top motifs enriched are labeled. D, AR binding compared with previously published E2F1 binding in the C4-2-shCon and C4-2-shRB models (Mandigo et al., 2021; ref. 19). Common binding is defined as binding overlap of one or more base pairs. Number of binding sites for AR and E2F1 are displayed for each model. Binding intensities are also shown (bottom).

models identified 3,976 AR/E2F1 overlapping binding sites, equivalent to $10.6 \%$ of total AR binding. Following RB depletion, the number of AR/E2F1 overlapping binding sites increased to 6,913, representing $19.9 \%$ of total AR binding (Fig. 1D). This nearly $10 \%$ increase in AR/ E2F1 co-occupancy after RB depletion suggests the strong potential for AR to affect E2F1 binding after RB loss.

\section{AR/E2F1 co-occupancy can be categorized into two distinct subgroups of binding events}

Further examination of AR/E2F1 co-occupancy after RB depletion identified two distinct subgroups of binding: (i) sites with AR and E2F1 co-occupancy in both the RB-intact and RB-depleted conditions (termed "constitutive co-occupancy binding" or CCB, 1,565 sites) and (ii) sites of AR gain to an existing E2F1-bound site, E2F1 gain to an existing AR-bound site, or concordant gained AR, and E2F1 binding (termed "gained co-occupancy binding" or GCB,
5,284 sites; Fig. 2A, left). Representative AR and E2F1 binding peaks were visualized using the Integrative Genomics Viewer and are shown along with ChIP-qPCR validation of AR/E2F1 cooccupancy in two distinct models of advanced disease (Fig. 2A, right; Supplementary Fig. S2A and S2B). To further explore regulatory element localization of these co-occupancy patterns, binding region annotation was performed. CCB was specific to promoter, distal intergenic, and intronic regions, all of which are known regions of either AR or E2F1 binding (Fig. 2B). Localization of $\mathrm{GCB}$ was almost exclusively found at distal intergenic and intronic regions of DNA (Fig. 2B). This specific localization pattern of GCB is expected, as it recapitulates the gained AR binding seen after RB depletion identified herein (Fig. 1B) and gained E2F1 binding upon $\mathrm{RB}$ depletion as previously described $(18,19)$. To examine the regions of GCB further, binding was examined based on type of gain: (i) sites that gained E2F1 binding, (ii) sites that gained AR 
A
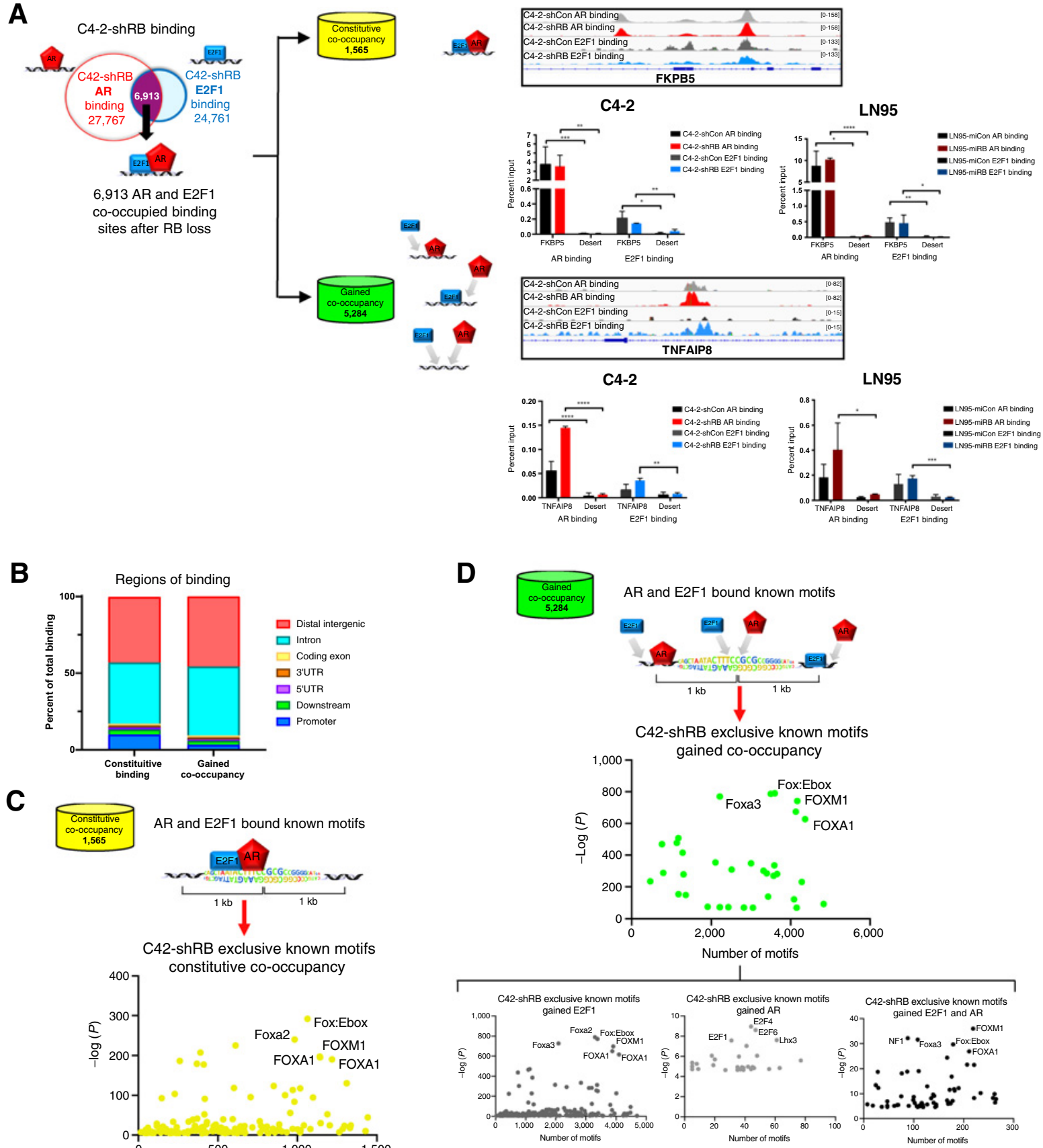

Figure 2.

AR and E2F1 co-occupancy can be defined by two subgroups of binding. A, AR and E2F1 co-occupancy after RB loss in C4-2-derived models based on mechanism of binding. Co-occupied sites were divided into two types of co-occupied binding: CCB (yellow) and GCB (green). The number of sites that fall under each type is shown (left). Representative binding tracing of AR and E2F1 for each type of binding is displayed, with ChIP-qPCR validation in C4-2 and LN95-derived models (right). B, Regions of binding for CCB and GCB were analyzed using the cis-regulatory element annotation system (CEAS) package. Regions are represented as a percentage of total binding. C, Known motif analysis within a 1-kb window from the center of E2F1/AR CCB. Top motifs enriched are labeled. D, Known motif analysis within a 1-kb window from the center of E2F1/AR GCB after RB1 depletion. Motif analysis was further evaluated by the mechanism of gained binding including gained E2F1, gained $\mathrm{AR}$, and gained both AR and E2F1 binding, respectively (bottom). Top motifs enriched are labeled. ${ }^{*}, P<0.05 ;{ }^{* *}, P<0.01 ;{ }^{* * *}, P<0.001 ;{ }^{* * *}, P<0.0001$.

binding, or (iii) sites that gained both $\mathrm{AR}$ and E2F1 binding. Analysis revealed that sites that gained E2F1 alone, or gained both E2F1 and AR binding, were almost exclusively at distal intergenic and intronic regions as expected. However, $50 \%$ of sites that gained
AR binding were at promoter regions (Supplementary Fig. S2C), suggesting that at these sites, AR is localized to regions where E2F1 is primarily positioned. These data provide evidence that, although regions of binding may be distinct between binding categories, a 
majority of AR and E2F1 binding occurs in regions known to be associated with individual AR and E2F1 binding.

Given that this AR/E2F1 co-occupied binding occurs within AR and E2F1-associated regions of DNA, the DNA sequences proximal to the co-occupied binding sites were assessed to gain further insight into the potential mechanism driving this co-occupancy via known motif analysis. CCB sites were enriched for known AR-associated motifs including FOXA1 and FOXM1 (Fig. 2C). Overall, GCB sites were also enriched for FOXA1 and FOXM1, again implying AR is interacting with known coregulators (Fig. 2D; Supplementary Fig. S2D), and suggesting that AR and/or AR cofactors may be contributing to E2F1 localization to these sites after RB loss. Interestingly, the subcategories of GCB exhibited distinct motif enrichment patterns. Although ARassociated motifs were enriched in gained $\mathrm{E} 2 \mathrm{~F} 1$ alone and gained $\mathrm{AR} /$ E2F1 binding subcategories, sites that gained AR binding after RB loss were enriched for E2F-related motifs including E2F1, E2F4, and E2F6, again suggesting that $\mathrm{AR}$ may also be driven to sites of $\mathrm{E} 2 \mathrm{~F} 1$ after $\mathrm{RB}$ depletion (Fig. 2D, bottom). Together, these data reveal that a majority of AR/E2F1 co-occupancy is associated with AR-related motifs in distal intergenic and intronic regions, whereas sites exhibiting gained AR binding suggests potential cooperation with $\mathrm{E} 2 \mathrm{~F} 1$ at promoter regions. Although binding localization and motif analysis suggest increased site-specific AR/E2F1 interaction after RB loss, AR and E2F1 codependency at these co-occupied sites required further exploration.

\section{AR and E2F1 binding at co-occupied sites are codependent}

Given that the global increase of AR/E2F1 co-occupancy after RB depletion is driven by a rewiring of both $\mathrm{AR}$ and $\mathrm{E} 2 \mathrm{~F} 1$ binding in the presence of androgen stimulation, the dependence on AR activation to promote binding at these co-occupied sites was examined. AR and E2F1 binding at CCB sites and GCB sites was assessed with or without androgen stimulation. Supporting previous studies (21), AR and E2F1 binding was observed after three hours of androgen stimulation, whereas there was no significant AR or E2F1 binding at either the CCB or GCB sites under androgen deprivation (Fig. 3A), indicating that both AR and E2F1 binding to these co-occupied sites after RB depletion are reliant on AR activation. This dependence on androgen stimulation was further elucidated through examination of AR and E2F1 binding at various time points of stimulation. The translocation of AR into the nucleus has been observed as early as 30 minutes following DHT treatment (34); thus, the binding of AR and E2F1 was examined at early timepoints post AR stimulation including $0,1,2$, and 3 hours. Interestingly, assessment of CCB sites revealed consistent binding of $\mathrm{AR}$ and $\mathrm{E} 2 \mathrm{~F} 1$ across multiple timepoints between the $\mathrm{RB}$ depletion and control models with significant changes in E2F1 binding occurring primarily after two hours (Fig. 3B). These data reveal that E2F1 requires three hours of AR stimulation before binding to $\mathrm{CCB}$ sites, whereas AR binding occurs earlier, suggesting that $A R$ or $A R$ cofactors may be recruiting E2F1 to these sites after AR stimulation. Additionally, the lack of difference in $\mathrm{AR}$ and $\mathrm{E} 2 \mathrm{~F} 1$ binding before and after $\mathrm{RB}$ depletion indicates that $\mathrm{RB}$ has minimal effects on this constitutive binding. Binding examined at sites of GCB identified a clear separation between the RB intact and RB-depleted models revealing a gain in both $\mathrm{AR}$ and E2F1 binding exclusively after $\mathrm{RB}$ depletion as early as one-hour after treatment (Fig. 3C). These data suggest simultaneous recruitment of both AR and E2F1 exclusively after RB depletion, which may be driven by AR cofactors as predicted by the motif analysis. Overall, these data identify distinct binding kinetics between $\mathrm{CCB}$ and $\mathrm{GCB}$, reliant on androgen stimulation and $\mathrm{RB}$ depletion, and highlight a greater impact of RB depletion on GCB.
Given that E2F1 appears to bind either simultaneously with AR, or post-AR binding at co-occupied sites, the dependence of E2F1 binding on the presence of AR was assessed. To examine this, E2F1 binding was investigated after knockdown of AR. E2F1 binding was diminished following AR knockdown (Fig. 3D), whereas E2F1 protein expression remained unchanged. This loss of binding implies that E2F1 binding to these co-occupied sites after RB depletion is dependent on the presence of AR (Fig. 3D). Interestingly, when AR binding was assessed in response to E2F1 knockdown, a similar result was observed. AR binding was significantly decreased in response to E2F1 depletion (Fig. 3E). However, AR protein expression was also significantly reduced with E2F1 knockdown, suggesting that this dependence may be driven by E2F1 transcriptional control of AR, instead of recruitment of AR protein to DNA by E2F1. Overall, these data indicate that AR/ E2F1 co-occupancy after RB depletion is reliant on E2F1 transcription of $A R$ and the activation of AR signaling.

\section{AR and E2F1 coregulate transcription after RB depletion}

Given that RB depletion drives an increase in AR/E2F1 cooccupancy on DNA and binding to these co-occupied sites requires the function of E2F1 and active AR, the biological significance of this binding was assessed through transcriptional studies. Genes with transcriptional start sites (TSS) within $30 \mathrm{~kb}$ of validated AR/E2F1 co-occupied binding were selected as putative targets of AR and E2F1 coregulation. Expression of genes with CCB (FKBP5, SGK1, and FZD1), and those with GCB (TNFAIP8, ESRP1, and FOXN3), was examined after modulation of $\mathrm{AR}$ and $\mathrm{E} 2 \mathrm{~F} 1 \mathrm{in} \mathrm{RB}$ intact and depleted conditions (Fig. 4A). The expression of CCB genes was significantly increased from 1.5- to 50-fold following androgen stimulation with DHT (Fig. 4A; Supplementary Fig. S3A, black). Expression of these genes was further increased $>1.5$-fold after RB depletion (Fig. 4A; Supplementary Fig. S3A, C4-2: red, LN95: dark red). These expression changes indicate that transcription of these genes is AR dependent and increased after RB depletion. Further, modulation of E2F1 through transient E2F1 knockdown led to a $>1.3$-fold decrease in gene expression (Fig. 4A; Supplementary Fig. S3A, C4-2: blue, LN95: gray), indicating that transcription of these genes is also regulated by E2F1. Similar trends were observed when genes with GCB were examined. Here, a $>1.5$-fold increase in expression was observed after androgen stimulation and RB depletion. Following modulation of E2F1, gene expression was decreased >1.5-fold (Fig. 4B; Supplementary Fig. S3B). These data support a cooperation between AR and E2F1 in the regulation of gene expression after RB depletion. Importantly, these trends in gene expression changes were translated to the protein level, where similar changes in protein expression were observed, a $>1.3$-fold increase in response AR stimulation, a $>1.2$-fold increase after RB depletion, and a significant 1.1-fold decrease after E2F1 modulation (Fig. 4C). Overall, assessment of these representative genes reveals that AR/E2F1 co-occupancy leads to the enhanced coregulation of gene transcription after RB loss, that is further translated to increased protein expression, suggesting that $\mathrm{AR}$ and E2F1 coregulation after RB depletion may have a significant impact on tumor biology.

To examine the biological impact of AR and E2F1 transcriptional coregulation on a global level, genes with TSSs within $30 \mathrm{~kb}$ of the 6,913 $\mathrm{AR} / \mathrm{E} 2 \mathrm{~F} 1 \mathrm{co}-\mathrm{occupied}$ binding sites were identified as putative AR and E2F1 coregulated targets. This method identified 1,272 genes with CCB and 3,195 genes with GCB (Fig. 4D). To select for coregulated genes that are likely to have a biological impact, the genes were compared with previously published RNA-seq after RB depletion under androgen stimulation (19). This comparison led to the 
A
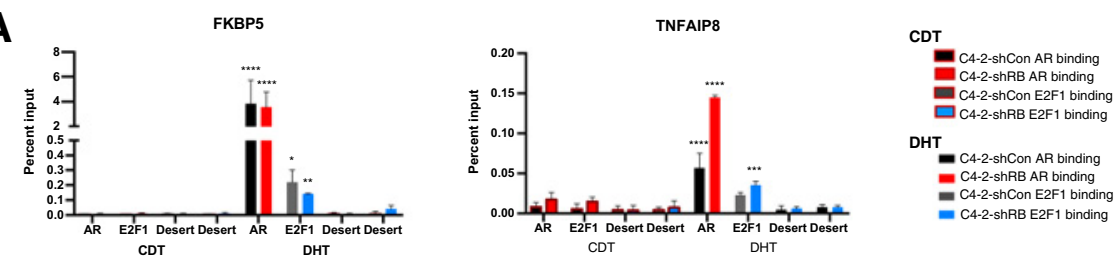

B
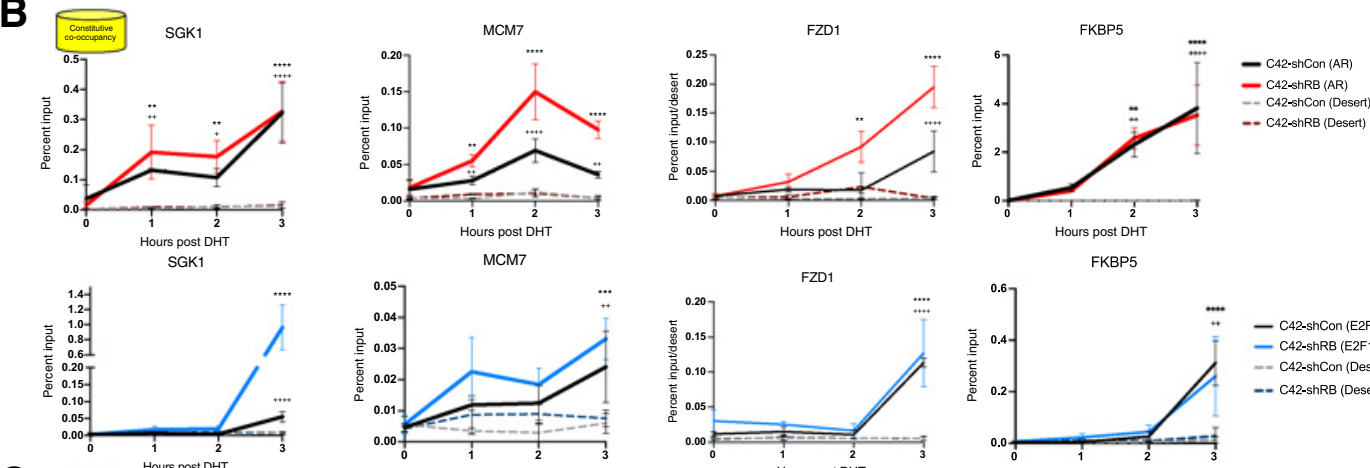

C
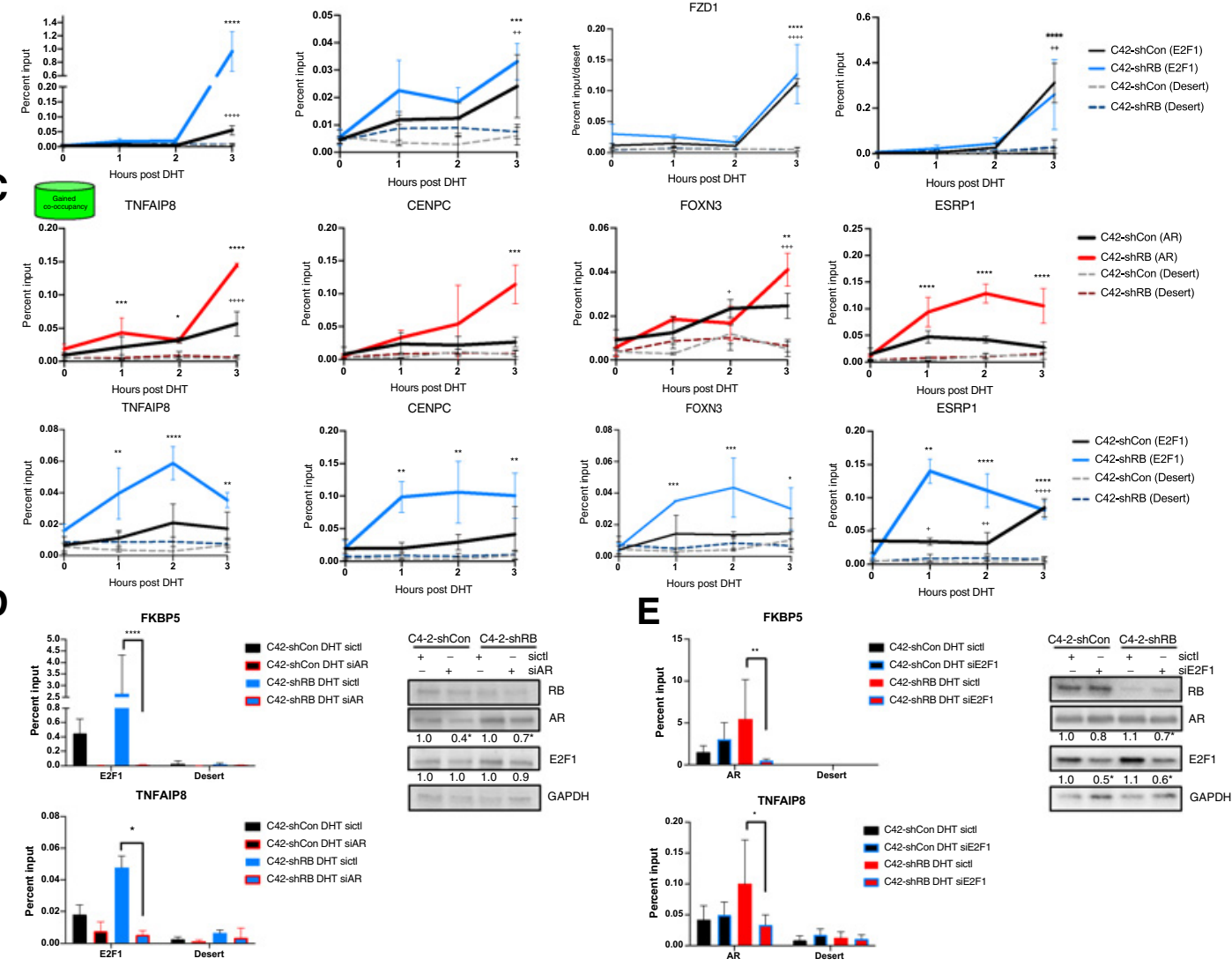

Figure 3.

AR and E2F1 binding at co-occupied sites is codependent. A, AR and E2F1 binding in C4-2-shCon and C4-2-shRB models after 72 hours androgen depletion and 3-hour $10 \mathrm{nmol} / \mathrm{L} \mathrm{DHT}$-treated conditions. B, AR and E2F1 CCB at increasing time points (0, 1, 2, 3 hours) following $10 \mathrm{nmol} / \mathrm{L}$ of DHT treatment in C4-2-shCon and C4-2-shRB models. Significance is defined by ${ }^{+}$for C4-2-shCon and * for C4-2-shRB. C, AR and E2F1 GCB at increasing time points ( $0,1,2,3$ hours) following $10 \mathrm{nmol} / \mathrm{L}$ of DHT treatment in C4-2-shCon and C4-2-shRB models. D, E2F1 binding following 72 hours of AR transient knockdown and 3 hours of $10 \mathrm{nmol} / \mathrm{L}$ DHT treatment in C4-2shCon and C4-2-shRB models. Immunoblot validating knockdown protein is shown with quantification. E, AR binding following 72 hours of E2F1 transient knockdown and 3 hours of $10 \mathrm{nmol} / \mathrm{L} \mathrm{DHT}$ treatment in C4-2-shCon and C4-2-shRB models. Immunoblot is shown with quantification. Significance is displayed for each cell line as siE2F1/siAR compared with sictl; * $P<0.05 ;{ }^{* *}, P<0.01 ;{ }^{* * *}, P<0.001 ;{ }^{* * * *}, P<0.0001$.

identification of 199 genes associated with CCB and 419 genes associated with GCB that displayed increased expression after RB loss (Fig. 4E). Identified genes were further assessed through Gene Set Enrichment Analysis. Enriched pathways for genes with CCB included pathways for androgen response and E2F targets, indicating that AR and E2F1 coregulate canonical androgen-regulated pathways as pre- viously shown (21), as well as additional pathways known to be associated with RB loss and disease progression including hypoxia, $\mathrm{G}_{2}-\mathrm{M}$ checkpoint, and metabolic signaling. Enriched pathways for genes with GCB included androgen response, $\mathrm{G}_{2}-\mathrm{M}$ checkpoint, and metabolic signaling in addition to pathways not previously associated with $\mathrm{RB}$ transcriptional control such as TNF $\alpha$ signaling, IL6 JAK 
A

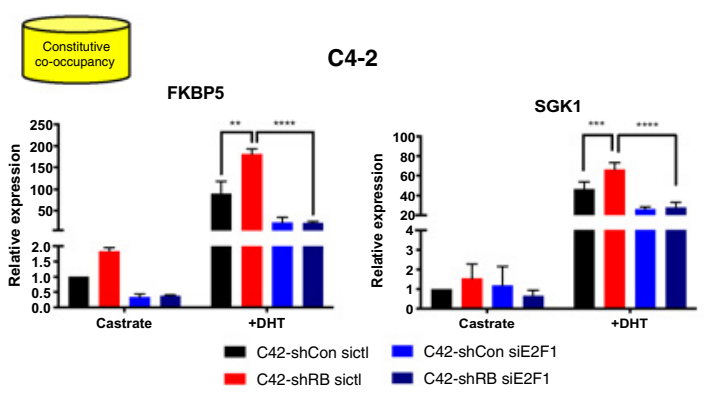

B
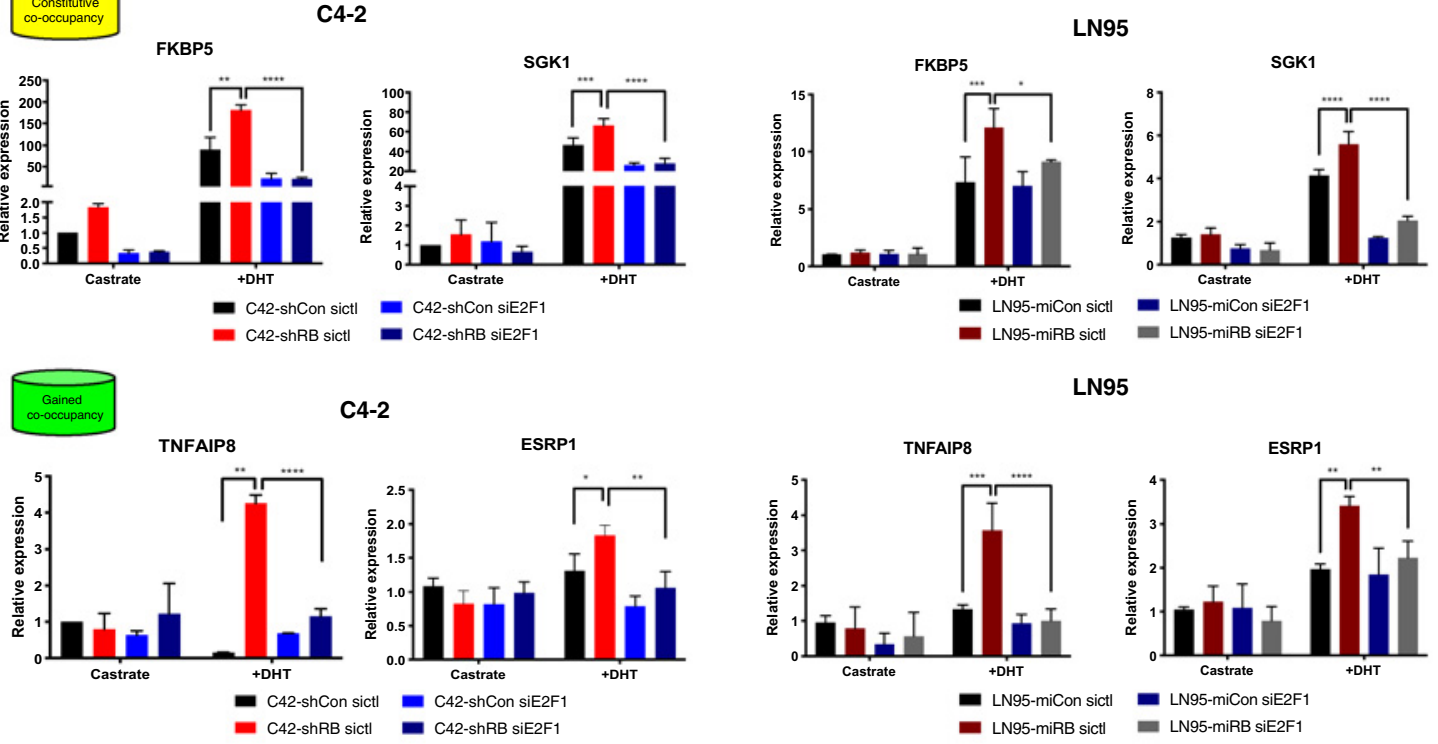

LN95

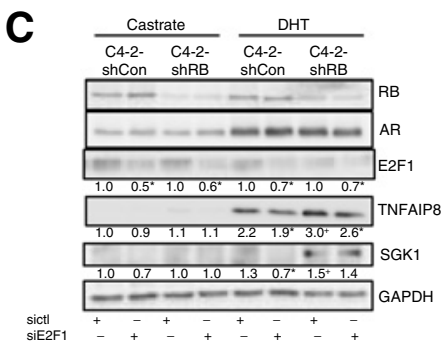

E
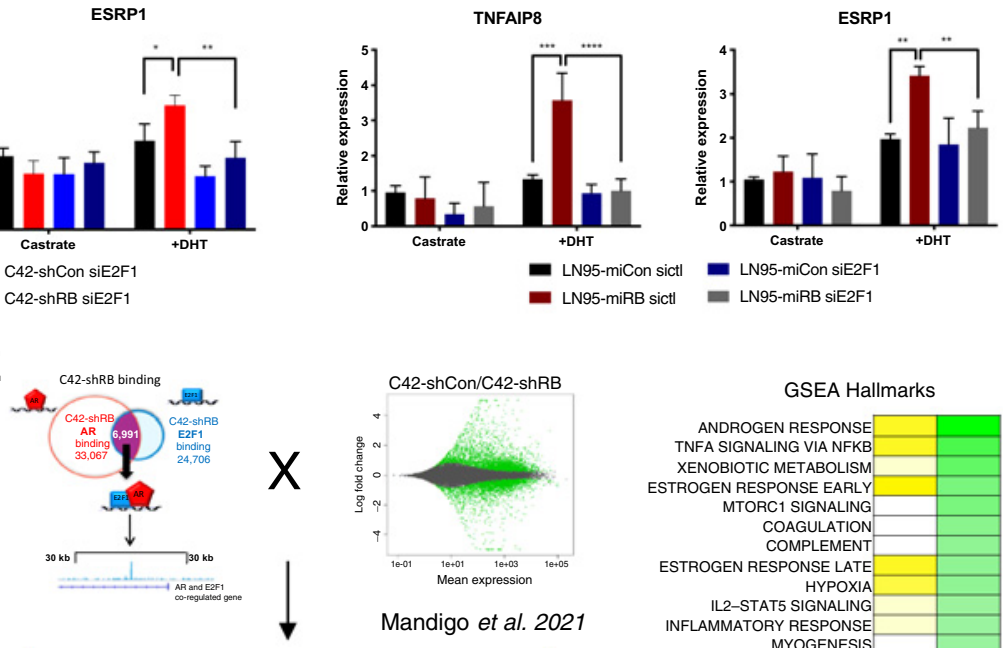

D

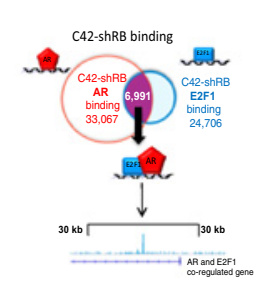

Genes with co-occupied AR and E2F1 binding
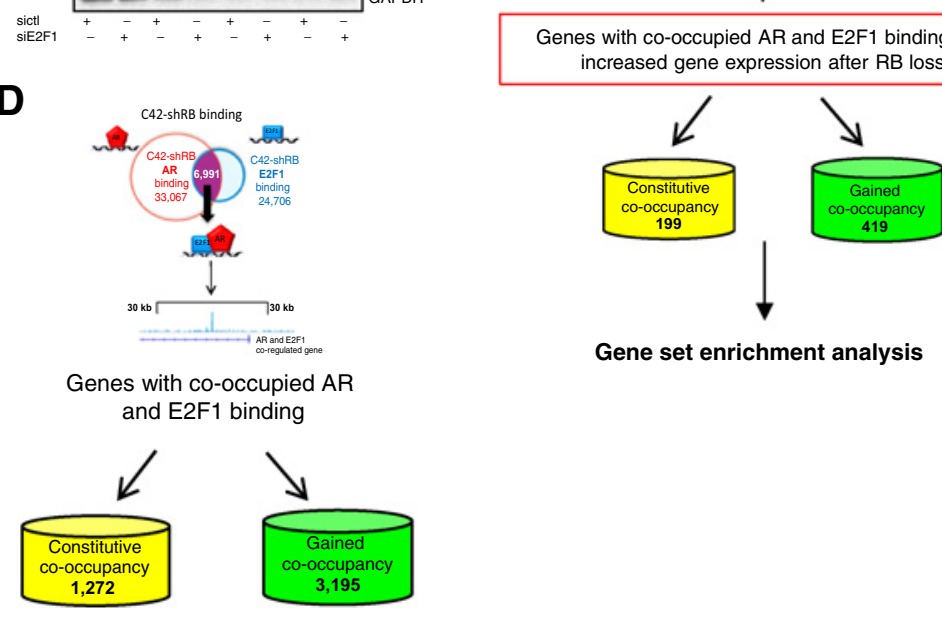

Gene set enrichment analysis

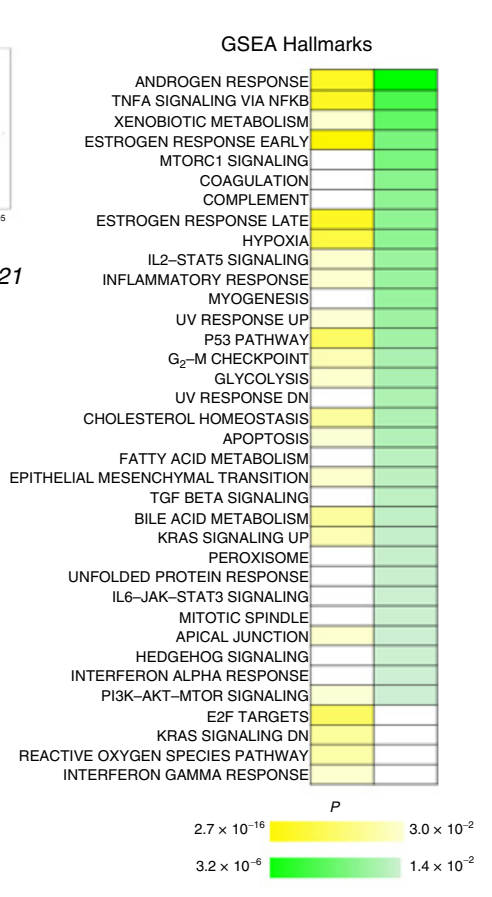

Figure 4.

AR and E2F1 coregulate transcription after RB depletion in CRPC. A and B, Transcriptional analysis of select genes with co-occupied AR/E2F1 binding from each subgroup of co-occupancy in C4-2 and LN95-derived models. Cells were deprived of androgens for 72 hours and harvested (castrate) or treated with 10 nmol/L DHT for 16 hours (DHT) and treated with either scrambled siRNA (sictl) or siE2F1. C, Protein expression is shown after 24 hours (DHT) in C4-2-shCon and C4-2-shRB models. D, AR/E2F1 co-occupied binding after RB depletion in C4-2-derived models was mapped to potential target genes with TSS within $30 \mathrm{~kb}$ of the co-occupied binding site. Genes were categorized by mechanism of binding. E, Genes with an AR/E2F1 co-occupied binding site with $30 \mathrm{~kb}$ of the TSS were overlapped with genes altered from previously published RNA-seq after RB depletion (Mandigo et al., 2021; ref. 19). GSEA Hallmark pathway analysis was performed based on subgroup of binding. Pathways are shown. Significance is displayed for each cell line as siE2F1 compared with sictl; ${ }^{*}, P<0.05 ;{ }^{* *}, P<0.01 ;{ }^{* * *}, P<0.001 ;{ }^{* * * *}, P<0.0001$, or as shRB/miRB compared with shCon/miCon; ${ }^{+}, P<0.05 ;^{++}, P<0.01 ;^{+++}, P<0.001{ }^{++++}, P<0.0001$.

STAT3 signaling, and interferon alpha response, suggesting that AR and $\mathrm{E} 2 \mathrm{~F} 1$ are cooperating in this RB-depleted, late stage of disease, to regulate novel pathways associated with apoptosis and immune response.
These data reveal that $\mathrm{RB}$ depletion drives an increase in $\mathrm{AR}$ and E2F1 codependent co-occupancy on DNA that promotes the coregulation of genes associated with a vast number of cancer-related pathways. RB function has been previously associated with TNF $\alpha$-induced 
apoptosis beyond its role as a transcriptional regulator (35-37). The protein is cleaved by caspases promoting the downstream caspase signaling cascade and has been shown to translocate to the mitochondria where it binds with apoptosis-associated factors to induce mitochondrial membrane permeabilization and initiate apoptosis (38). However, the transcriptional control of apoptosis by RB and the consequence of $\mathrm{RB}$ loss on apoptosis, specifically through $\mathrm{AR}$ and E2F1 coregulation, have yet to be explored. To validate the biological significance of this coregulation on apoptosis response and disease progression, the impact of AR and E2F1 transcriptional control on TNF $\alpha$-signaling and apoptosis was further investigated.

\section{AR/E2F1 cooperation after RB depletion provides protection from apoptosis}

Tumor necrosis factor alpha-induced protein 8 (TNFAIP8) plays a key role in TNF $\alpha$ signaling and was identified herein as having gained $\mathrm{AR}$ and E2F1 co-occupied binding, and increased expression after RB depletion. It has been shown across multiple cancers to function as a prosurvival protein that protects against apoptosis through inhibition of caspase activation $(39,40)$. Previous studies reported induction of TNFAIP8 protein levels in response to androgen stimulation, linking TNFAIP8 to AR control and supporting the data herein $(41,42)$. To assess the biological impact of increased TNFAIP8 after RB depletion, apoptosis induced by TNF $\alpha$ in combination with actinomycin D to block transcription, was evaluated. The cleavage of known substrates of the caspase signaling cascade, poly (ADP-ribose) polymerase 1 (PARP1) and capsase-3 were utilized as a measurement of apoptosis $(43,44)$. When treated with TNF $\alpha$ and actinomycin D, levels of cleaved PARP and caspase-3, compared with total protein, were significantly decreased (2- and 1.4-fold, respectively) in the RBdepleted models (Fig. 5A). These data suggest a protective advantage of RB depletion against TNF $\alpha$-induced apoptosis. To challenge this postulate using clinically relevant cytotoxic therapy, apoptosis in response to cisplatin was also measured. Similarly, RB-depleted models showed a significant decrease in cleaved PARP and caspase- 3 compared with total protein expression (1.7 and 1.1-fold, respectively). These changes were validated in additional RB isogenic CRPC models showing a 1.3- and 1.7-fold decrease in PARP and caspase-3 cleavage, respectively (Fig. 5B). These data suggest that RB depletion drives a protective advantage against apoptosis-inducing therapies.

To assess whether the antiapoptotic effect of $\mathrm{RB}$ depletion is attributed to AR and E2F1 cooperation, PARP and caspase- 3 cleavage in response to TNF $\alpha$ was assessed after E2F1 knockdown and AR stimulation in RB-depleted models. Quantification of cleaved PARP and caspase- 3 demonstrated that these markers of apoptosis were significantly decreased following RB depletion; however, knockdown of E2F1 mitigated the effect of RB depletion on both cleaved PARP and caspase-3, with latter increased 1.4-fold after E2F1 knockdown (Fig. 5C). These data indicate that protection from apoptosis in response to AR stimulation and TNF $\alpha$ in combination with actinomycin D is directly regulated by E2F1 after RB depletion. To assess this regulation further, apoptosis was assessed after modulation of TNFAIP8. As shown, RB depletion induced a significant decrease in cleaved PARP and caspase- 3 in response to TNF $\alpha$ and actinomycin D under AR stimulation. However, this downregulation of apoptosis was reversed after knockdown of TNFAIP8 (Fig. 5D), demonstrating that $\mathrm{RB}$ depletion-induced protection from apoptosis is controlled by TNFAIP8. To assess further the impact of RB depletion on apoptosis, cell viability of CRPC isogenic models treated with actinomycin $D$ and varying concentrations of TNF $\alpha$ was assessed. RB-depleted models were treated consistently with $5 \mu \mathrm{g} / \mathrm{mL}$ of actinomycin D and varying concentrations of $\mathrm{TNF} \alpha(0.1$ to $50 \mathrm{ng} / \mu \mathrm{L})$. RB-depleted models showed a significantly 1.2 -fold lower response to treatment across all concentrations of TNF $\alpha$. This decreased sensitivity to TNF $\alpha$ was further validated in additional RB-depleted isogenic models where $\mathrm{RB}$ depletion led to a significant 1.1-fold lower response to TNF $\alpha$ at concentrations above $5 \mathrm{ng} / \mu \mathrm{L}$ (Fig. 5E), indicating that RB depletion renders cells less sensitive to TNF $\alpha$ treatment in combination with actinomycin $\mathrm{D}$. In conclusion, these data confirm a protective advantage of RB depletion from cytotoxic therapy through AR and E2F1 driven increase of TNFAIP8.

\section{Clinical relevance of AR/E2F1 coregulation}

Given the robust identification of AR and E2F1 coordinated function after RB loss (Figs. 2, 3, and 4) and the resultant impact on response to antitumor agents (Fig. 5), the expression of CCB and GCB genes was examined across RB-intact and RB-low tumor cohorts from the RMH. The expression of AR/E2F1 coregulated genes positively correlated with $E 2 F 1$ expression and AR activity in RB low tumors. Importantly, GCB genes including TNFAIP8, MCM7, FZD1, and TK1 showed stronger, significant, positive correlations between E2F1 expression and AR activity in the RB low tumors compared with RB intact (Fig. 6A; Supplementary Fig. S4A). As expected, positive correlations between E2F1 expression and AR activity, and the coregulated CCB genes including FKBP5, ESRP1, ZBTB10, and DUS4 L are present in the RB-intact and RB-low tumor cohorts (Fig. 6B; Supplementary Fig. S4B), as binding was constitutive before and after $\mathrm{RB}$ depletion. These data demonstrate that RB loss-induced AR and E2F1 coregulation of genes occurs clinically in advanced disease, suggesting that the biological implications driven by AR and E2F1 cooperation after RB loss identified herein are of significant translational relevance.

Together, these data reveal a potential regulatory mechanism of AR and E2F1 controlled transcription in which AR promotes expanded E2F1 binding after RB loss, and are the first to identify enhanced AR and $\mathrm{E} 2 \mathrm{~F} 1$ transcription coregulation in RB-deficient disease. This study identifies a protective advantage from apoptosis conferred by $\mathrm{RB}$ loss through $\mathrm{AR}$ and E2F1 transcriptional cooperation and reveals a vast number of clinically relevant genes associated with $\mathrm{AR}$ and $\mathrm{E} 2 \mathrm{~F} 1$ that are frequently altered in disease (Fig. 6C); overall implicating a biological role of $\mathrm{AR}$ and $\mathrm{E} 2 \mathrm{~F} 1$ coregulation in the progression of advanced disease.

\section{Discussion}

Although it is well established that RB function is frequently lost in cancer and promotes disease progression, the molecular mechanisms by which RB loss promotes aggressive features remain incompletely understood. The current study identifies the enhanced cooperation between two oncogenic transcriptional regulators as a consequence of $\mathrm{RB}$ loss, which contributes to tumor phenotypes. Key findings reveal that: (i) RB depletion elicits a shift in AR and E2F1 binding, driving an increase in co-occupancy on chromatin; (ii) AR/E2F1 co-occupancy is androgen dependent and relies on E2F1 function; (iii) AR and E2F1 cooperate to regulate novel transcriptional networks of cancer relevance after $\mathrm{RB}$ depletion; (iv) resultant AR/E2F1 regulation of TNFAIP8 induces a protective advantage against tumor cell death in RB-deficient disease; and (v) AR/E2F1 coregulated genes correlate with AR activity and E2F1 expression in human tumors. Taken together, these studies identify a clinically significant mechanism in which AR and E2F1 cooperate to protect RB-deficient disease from therapeutic intervention. 
A

C4-2

C4-2- C4-2- C4-2- C4-2- C4-2- C4-2shCon shRB shCon shRB shCon shRB $+\quad+\quad+\quad+\quad+\quad+10$ nmol/L DHT

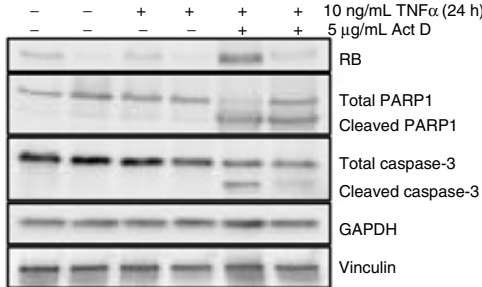

TNF $\alpha$ and Act D treatment

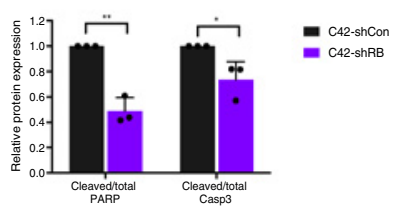

C

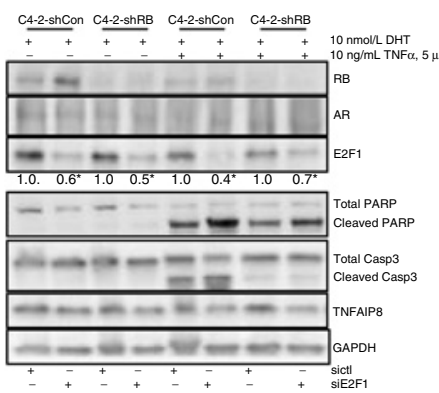

E
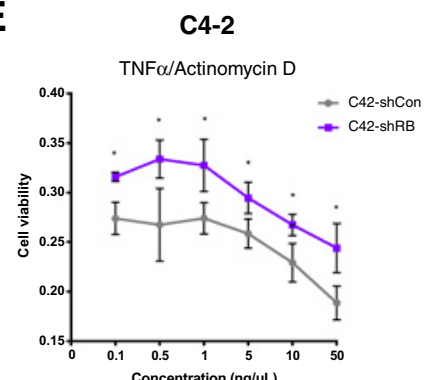

B

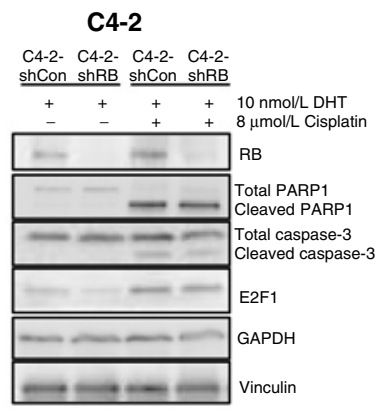

Cisplatin treatment

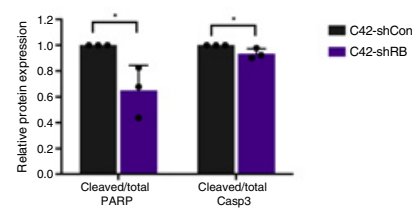

D

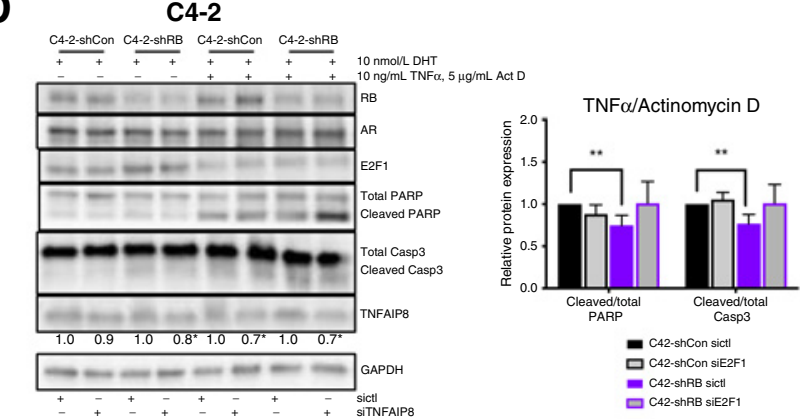

LN95

LN95- LN95- LN95- LN95miCon miRB miCon miRB
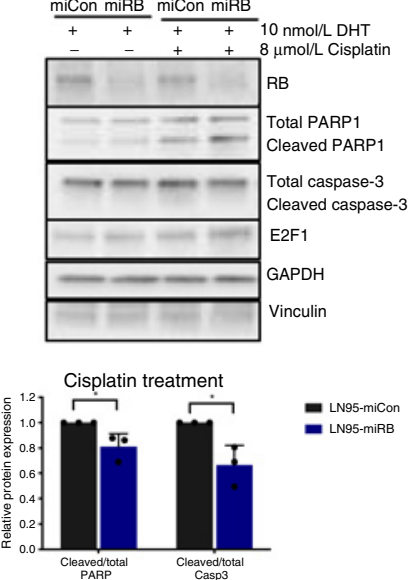

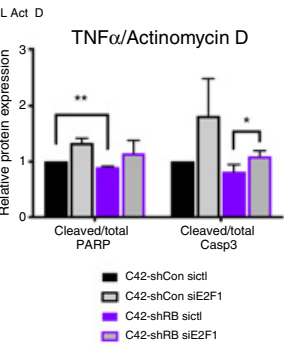

LN95

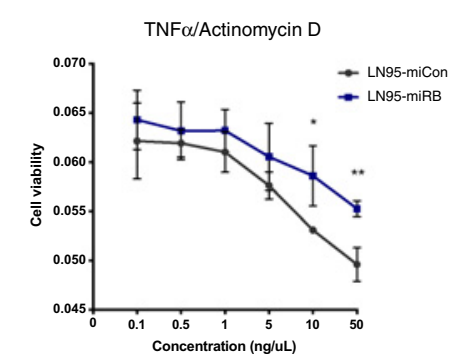

Figure 5.

RB depletion results in protection from TNF $\alpha$-induced apoptosis through AR/E2F1 coregulation of TNFAIP8. A, Western blot after treatment with 10 ng/ $\mu \mathrm{L}$ TNF $\alpha$ protein and $5 \mu \mathrm{g} / \mu \mathrm{L}$ actinomycin D in C4-2-shCon and C4-2-shRB models. Quantification of cleaved/total caspase-3 and PARP is shown. B, Western blot after treatment with $8 \mu \mathrm{mol} / \mathrm{L}$ cisplatin treatment in C4-2- and LN95-derived models of RB depletion. Quantification of cleaved/total caspase-3 and PARP is shown. C and D, Western blot after treatment with $10 \mathrm{ng} / \mu \mathrm{L}$ TNF $\alpha$ protein and $5 \mu \mathrm{g} / \mu \mathrm{L}$ actinomycin D treatment following 72 hours of E2F1 (C) and TNFAIP8 transient knockdown (D). C4-2-shCon and C4-2-shRB models were treated with 10 nmol/L DHT for 24 hours following 72 hours in CDT. Quantification of cleaved/total caspase-3 and PARP is shown. E, Cytotoxicity assay in C4-2- and LN95-derived models in response to treatment with varying concentrations of TNF $\alpha$ protein $(0.1-50 \mathrm{ng} / \mu \mathrm{L})$ and $5 \mu \mathrm{g} / \mu \mathrm{L}$ of actinomycin $\mathrm{D}$. Significance displayed below blots is relative to each cell line sictl: $*, P<0.05 ;{ }^{* *}, P<0.01$

The data herein reveal a shift in AR and E2F1 chromatin binding after $\mathrm{RB}$ depletion that promotes a striking increase in AR and E2F1 co-occupancy on DNA, providing the first genome-wide comparison of AR and E2F1 function in RB-deficient disease. Global assessment of $\mathrm{AR}$ and E2F1 binding revealed 6,913 overlapping binding sites after $\mathrm{RB}$ depletion, referred to as the AR/E2F1 cooperome, which is further distinguished as CCB or GCB (Figs. 1D and 2A). The concept that
E2F1 utilizes oncogenic cooperating transcriptional partners after RB loss opens the field to new areas of investigation as per the mechanisms of regulating this process. Previous studies revealed that RB loss has little impact on chromatin accessibility and that the overall chromatin landscape remains conserved even after androgen stimulation (18). These previous studies suggest that changes in access to chromatin after $\mathrm{RB}$ loss are not a main driver of increased AR and E2F1 
A
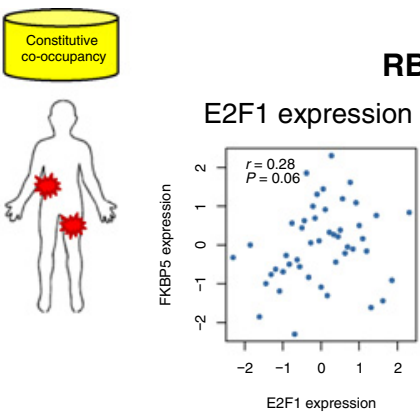

RB intact

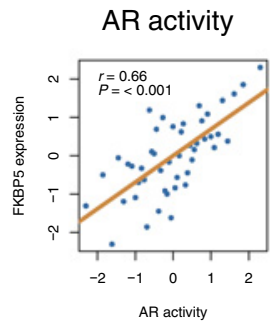

\section{Metastatic CRPC}

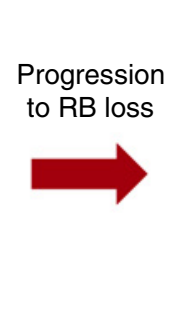

Metastatic CRPC

\section{RB intact}
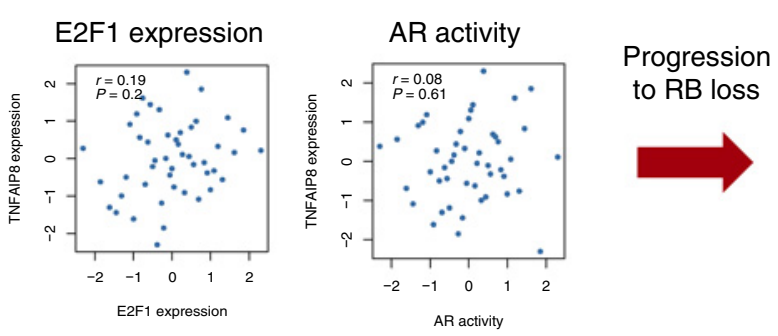

RB low
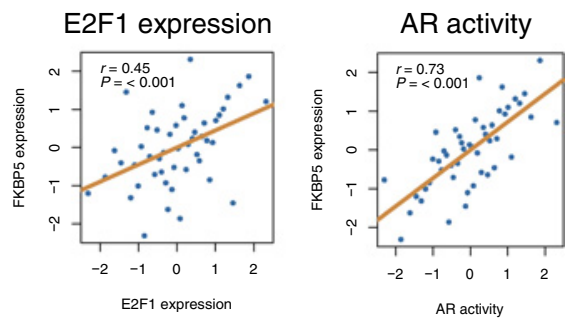

B

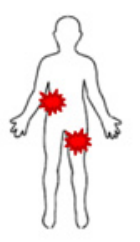

C

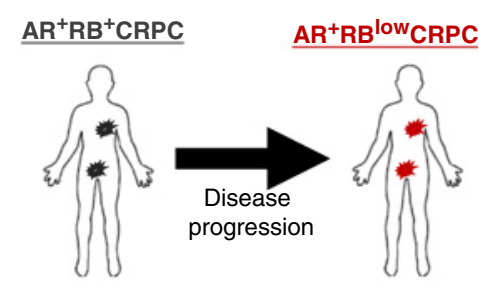

RB intact

RB deplete

Increased AR/E2F1 DNA co-occupancy
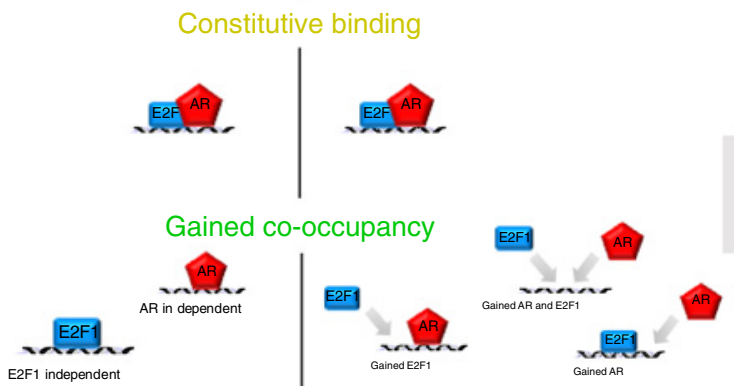

- AR and E2F1 coregulation of transcription

- Cooperation to drive advanced disease

- Protection from apoptosis

Resistance to cytotoxic

therapy

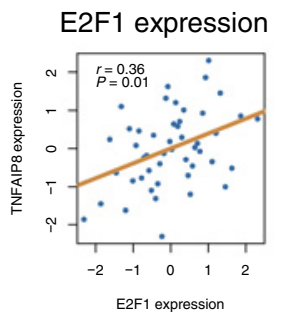

RB low

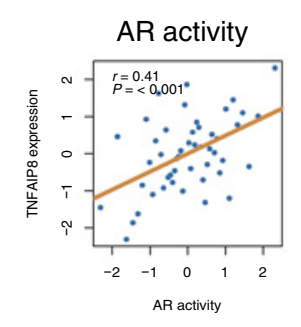

Figure 6.

AR/E2F1 coregulated genes correlate with AR activity and E2F1 expression in RB deplete tumors. RNA sequencing from fresh tumor biopsies was performed on patient samples from a novel metastatic CRPC patient cohort from RMH in London, UK $(n=98)$. The cohort was divided into RB-intact and RB-deplete tumors. A, The expression of FKBP5 (CCB) was examined and compared with expression of E2F1 and activity of AR. A positive correlation was observed when the expression of FKBP5 was compared with the expression of E2F1 in the RB-low tumors $(r=4.5, P<0.001)$. When compared with AR activity, a stronger positive correlation between $F K B P 5$ expression and AR activity was observed in the RB-low tumors (RB intact: $r=0.66, P<0.001$; RB low: $r=0.73, P<0.001)$. B, The expression of TNFAIP8 (GCB) was also compared with E2F1 expression. Positive correlations between TNFAIP8 and E2F1 expression, and the expression of TNFAIP8 and AR activity, were only observed in RB low tumors (E2F1 expression: $r=0.36, P=0.01 ;$ AR activity: $r=0.41$, $P<0.001$ ). C, RB depletion drives an increase in AR and E2F1 co-occupancy on DNA and coregulation of transcription. This co-occupancy can be divided by those sites that gained binding and those that maintained binding before and after RB loss. This co-occupancy promotes AR and E2F1 coregulated transcription, providing a protection from TNF $\alpha$-induced apoptosis. 
co-occupancy. Beyond the chromatin landscape, protein-protein interactions have a significant role in regulating transcription. Previous studies have confirmed that AR and E2F1 physically interact on chromatin, implying that AR and E2F1 bind either directly to each other or in a complex with additional factors (21). Interestingly, known and de novo motif analysis revealed the presence of FOX-associated motifs including FOXA1, FOXM1, and Fox:Ebox in proximity to AR/ E2F1 co-occupied binding, implicating the presence of AR cofactors at these sites (Fig. 2C and D). FOX proteins have been shown to play a significant role in prostate cancer initiation and development $(31,45)$ and are elevated in metastatic prostate cancer tumors (46). Although these factors are well described to bind AR, studies have also implicated a role for them in E2F1-dependent transcription showing that knockdown of FOXA1 results in a decrease in $\mathrm{E} 2 \mathrm{~F} 1$ protein expression as well as E2F1 target gene expression including CCND1 and CDK1 (47). Further, FOXM1 has been shown to directly interact with AR to regulate the expression of CDC6, a known target of E2F1-regulated transcription $(48,49)$. Comparison of previously published FOXA1 and FOXM1 cistromes $(50,51)$ with the AR/E2F1 cooperome revealed a greater than 3-fold increase in the number of FOXA1 and FOXM1 binding sites that overlapped with $\mathrm{AR} / \mathrm{E} 2 \mathrm{~F} 1$ binding after $\mathrm{RB}$ depletion. Overall, the enrichment of FOXA1 and FOXM1 motifs in proximity to AR/E2F1 co-occupied sites after RB loss, combined with this observed increase in FOXA1 and FOXM1 binding overlap, and previous data showing direct interaction between FOXA1 and FOXM1 with AR suggest that AR and E2F1 may be binding in complex with these cofactors after RB depletion. Together, these data are the first to reveal that $\mathrm{RB}$ depletion in advanced disease drives increased $A R$ and E2F1 co-occupancy and nominate FOX proteins as putative effectors of the resulting oncogenic networks.

The clinical significance of RB loss has been described through alterations of the $R B 1$ gene and changes in downstream transcriptional control. Previous studies have utilized the transcriptional changes resulting from $\mathrm{RB}$ depletion as markers of therapy resistance and predictors of patient outcome through the use of gene expression signatures. These signatures have since become a staple in the clinical assessment of RB status. Critically, the studies herein identify that RB depletion promotes a protective advantage against apoptosis induction through AR/E2F1 coregulation of TNFAIP8. The biological significance of elevated TNFAIP8 expression was explored herein (Fig. 5), highlighting the importance of delineating the clinical significance of AR/E2F1 coregulated gene networks in the future. Genes with constitutive AR and E2F1 co-occupancy that were the most frequently altered in a metastatic prostate cancer cohort included ATAD2, TPD52, and TK1 (52), all of which have been previously associated with aggressive disease. ATAD2, which was also previously identified as an AR/E2F1 target gene in hormone-sensitive prostate cancer (21), plays a role in cell proliferation, migration, invasion, and apoptosis and has been targeted as part of an anticancer treatment (53). Previous studies revealed that through association with acetylated histones, ATAD2 functions as a pioneer factor for E2F1 (54). Studies have also shown that ATAD2 directly binds to AR, enhancing its transcriptional activity (55). These previous studies, combined with the identification of ATAD2 as a putative target of AR/E2F1 coregulated transcription, suggest that ATAD2 may function as part of a positive feedback loop, driving increased AR and E2F1 co-occupancy after RB depletion. Validation of this positive feedback loop between AR/E2F1 regulation and ATAD2 function may provide a novel avenue to clinically target advanced, RB-depleted CRPC. Further, elevated TPD52 transcript expression has been observed in high-grade prostate cancer tumors when compared with low-grade tumors, and amplification of the
TPD52 gene is associated with early prostate cancer-specific mortality $(56,57)$. TPD52-targeted therapy is also currently under investigation in vivo through use of anticancer vaccines (57), providing an additional potential method of targeting this late-stage disease. Moreover, TK1 or thymidine kinase 1, a known RB/E2F1 target gene, plays a significant role in nucleotide biosynthesis and has been previously shown as a clinically valuable biomarker in human malignancies (58), thus may be useful to detect RB-deficient CRPC tumors. Additionally, frequently altered genes that exhibited gained AR and E2F1 cooccupancy after RB depletion such as ESRP1, and APEX2 have also been shown to be highly expressed in human malignancies and have been implicated in epithelial-mesenchymal transition and DNA repair $(59,60)$, indicating that $\mathrm{AR} / \mathrm{E} 2 \mathrm{~F} 1$ cooperation may be contributing to advanced disease via a multitude of cancer-associated pathways. Taken together, the discovery that these drivers of advanced disease and potential druggable targets are induced as a function of AR/ E2F1 cooperation provides an initial understanding of how these pathways are regulated in human malignancies.

The findings herein shift current understanding of E2F1 function and introduce the novel concept that E2F1 cooperates with oncogenic transcription factors after $\mathrm{RB}$ loss to promote aggressive phenotypes. Although the study herein focuses on E2F1 cooperation with $\mathrm{AR}$, the concept of a collaboration between E2F1 and additional transcription factors has been previously suggested in malignant disease. Prior studies examining E2F1 binding after RB depletion in prostate cancer have identified potential coregulators of E2F1-dependent transcription through motif analysis. Motifs including GRE and Tlx were enriched proximal to novel E2F1 binding after RB loss, suggesting that E2F1 may cooperate with the glucocorticoid steroid receptor (GR) and the orphan receptor TLX (18, 19). E2F1-regulated transcription has been previously associated with GR function through downstream signaling in response GR activation (61). Although this study implicates a role for GR signaling in E2F1 function, the prospect of E2F1 and GR coregulating transcription is of future interest as it may occur in parallel to E2F1 cooperation with AR. Further, as the TLX motif is also enriched with E2F1 binding, the cooperation between E2F1 and nuclear receptor transcription may occur more frequently than previously expected and may extend beyond the nuclear receptors discussed herein. These previous studies expand on the current understanding of E2F1 function, specifically in cooperation with nuclear receptor-controlled transcription. Although E2F1 cooperation with AR is explored herein, the overall role of E2F1 in regulating transcription in the presence and absence of $\mathrm{RB}$ is still not fully understood.

On balance, this study sheds new light into the role of E2F1 as a tumor promoting factor through transcription factor cooperation and highlights an unexpected consequence of RB loss in human cancers. Further, this study elucidates a direct impact of RB loss on AR function, providing the first genome-wide assessment of $A R$ in response to $R B$ depletion. Taken together, current findings provide critical insight into the networks that drive disease progression and nominate novel therapeutic targets of RB-deficient disease.

\section{Authors' Disclosures}

J.J. McCann reports grants from NIH during the conduct of the study. W. Yuan reports grants from Jilin Huarui Gene Technology outside the submitted work. J.S. de Bono reports grants, personal fees, and nonfinancial support from AstraZeneca, grants, personal fees, and nonfinancial support from Genentech/Roche, personal fees from Novartis, personal fees and nonfinancial support from Daiichi Sankyo, grants and nonfinancial support from Harpoon Therapeutics, Immunic, Crescendo 
Therapeutics, grants and personal fees from Taiho and Merck Serono, personal fees from Bayer and MSD outside the submitted work. K.E. Knudsen reports consultant and/or advisory roles with Janssen, CellCentric, and Genentech in the last 36 months. No disclosures were reported by the other authors.

\section{Authors' Contributions}

A.C. Mandigo: Conceptualization, data curation, formal analysis, funding acquisition, validation, investigation, visualization, methodology, writing-original draft, writing-review and editing. A.A. Shafi: Conceptualization, writing-review and editing. J.J. McCann: Funding acquisition, investigation, writing-review and editing. W. Yuan: Formal analysis. T.S. Laufer: Investigation, writing-review and editing. D. Bogdan: Formal analysis. L. Gallagher: Formal analysis. E. Dylgjeri: Writingreview and editing. G. Semenova: Validation, writing-review and editing. I.A. Vasilevskaya: Writing-original draft, writing-review and editing. M.J. Schiewer Writing-review and editing. C. McNair: Software, formal analysis, methodology writing-original draft, writing-review and editing. J.S. de Bono: Formal analysis. K.E. Knudsen: Conceptualization, resources, supervision, funding acquisition, writing-original draft, project administration, writing-review and editing.

\section{References}

1. Dyson NJ. RB1: a prototype tumor suppressor and an enigma. Genes Dev 2016; 30:1492-502.

2. van den Heuvel S, Dyson NJ. Conserved functions of the pRB and E2F families. Nat Rev Mol Cell Biol 2008;9:713-24.

3. Jarrard DF, Modder J, Fadden P, Fu V, Sebree L, Heisey D, et al. Alterations in the p16/pRb cell cycle checkpoint occur commonly in primary and metastatic human prostate cancer. Cancer Lett 2002;185:191-9.

4. Brooks JD, Steven Bova G, Isaacs WB. Allelic loss of the retinoblastoma gene in primary human prostatic adenocarcinomas. Prostate 1995;26:35-9.

5. Ittmann MM, Wieczorek R. Alterations of the retinoblastoma gene in clinically localized, stage B prostate adenocarcinomas. Hum Pathol 1996;27:28-34

6. Cooney KA, Wetzel JC, Merajver SD, Macoska JA, Singleton TP, Wojno KJ. Distinct regions of allelic loss on 13q in prostate cancer. Cancer Res 1996;56 1142-5.

7. Tricoli JV, Gumerlock PH, Yao JL, Chi S-G, D'Souza SA, Nestok BR, et al. Alterations of the retinoblastoma gene in human prostate adenocarcinoma Genes Chromosomes Cancer 1996;15:108-14.

8. Herschkowitz JI, He X, Fan C, Perou CM. The functional loss of the retinoblastoma tumour suppressor is a common event in basal-like and luminal B breast carcinomas. Breast Cancer Res 2008;10:R75.

9. Yokota J, Wada M, Shimosato Y, Terada M, Sugimura T. Loss of heterozygosity on chromosomes 3, 13, and 17 in small-cell carcinoma and on chromosome 3 in adenocarcinoma of the lung. Proc Natl Acad Sci U S A 1987;84:9252-6.

10. Feugeas O, Guriec N, Babin-Boilletot A, Marcellin L, Simon P, Babin S, et al. Loss of heterozygosity of the $\mathrm{RB}$ gene is a poor prognostic factor in patients with osteosarcoma. J Clin Oncol 1996;14:467-72.

11. Patiño-García A, Piñeiro ES, Díez MZ, Iturriagagoitia LG, Klüssmann FA Ariznabarreta LS. Genetic and epigenetic alterations of the cell cycle regulators and tumor suppressor genes in pediatric osteosarcomas. J Pediatr Hematol Oncol 2003;25:362-7.

12. Zojer N, Königsberg R, Ackermann J, Fritz E, Dallinger S, Krömer E, et al. Deletion of 13q14 remains an independent adverse prognostic variable in multiple myeloma despite its frequent detection by interphase fluorescence in situ hybridization. Blood 2000;95:1925-30.

13. $\mathrm{Xu} \mathrm{H}, \mathrm{Xu} \mathrm{K}, \mathrm{He} \mathrm{HH}$, Zang C, Chen $\mathrm{C}-\mathrm{H}$, Chen $\mathrm{Y}$, et al. Integrative analysis reveals the transcriptional collaboration between EZH2 and E2F1 in the regulation of cancer-related gene expression HHS public access. Mol Cancer Res 2016;14:163-72.

14. Xu HJ, Cagle PT, Hu SX, Li J, Benedict WF. Altered retinoblastoma and p53 protein status in non-small cell carcinoma of the lung: potential synergistic effects on prognosis. Clin Cancer Res 1996;2:1169-76.

15. Cordon-Cardo C, Wartinger D, Petrylak D, Dalbagni G, Fair WR, Fuks Z, et al. Altered expression of the retinoblastoma gene product: prognostic indicator in bladder cancer. J Natl Cancer Inst 1992;84:1251-6.

16. Chen WS, Alshalalfa M, Zhao SG, Liu Y, Mahal BA, Quigley DA, et al. Novel Rb1loss transcriptomic signature is associated with poor clinical outcomes across cancer types. Clin Cancer Res 2019;25:4290-9.

\section{Acknowledgments}

This work was supported by NIH T32 grant to A.C. Mandigo (GM100836) NIH R01 grants to K.E. Knudsen (5R01CA17640105, 5R01CA18256905, and 5R01CA21732903), NCI F99 grant to J.J. McCann (F99CA212225), and the Sidney Kimmel Cancer Center (SKCC) Support Grant (5P30CA056036). Additional suppor was provided by the SKCC Cancer Genomics, Translational Research/Pathology and Biostatistics core services. The authors thank Dr. Jun Lou (John Hopkins University) for providing the LnCaP95 cells utilized in this study. The sponsors were critical in study design, data collection, analysis, interpretation, and review of the manuscript.

The costs of publication of this article were defrayed in part by the payment of page charges. This article must therefore be hereby marked advertisement in accordance with 18 U.S.C. Section 1734 solely to indicate this fact.

Received April 14, 2021; revised July 14, 2021; accepted September 9, 2021; published first October 8, 2021

17. Cecchini MJ, Ishak CA, Passos DT, Warner A, Palma DA, Howlett CJ, et al. Loss of the retinoblastoma tumor suppressor correlates with improved outcome in patients with lung adenocarcinoma treated with surgery and chemotherapy. Hum Pathol 2015;46:1922-34

18. McNair C, Xu K, Mandigo AC, Benelli M, Leiby B, Rodrigues D, et al. Differential impact of RB status on E2F1 reprogramming in human cancer. J Clin Invest 2018;128:341-58

19. Mandigo AC, Yuan W, Xu K, Gallagher P, Pang AYF, Guan YFAA, et al. RB/ E2F1 as a master regulator of cancer cell metabolism in advanced disease. Cancer Discov 2021;11:2334-53.

20. Sharma A, Yeow W-S, Ertel A, Coleman I, Clegg N, Thangavel C, et al. The retinoblastoma tumor suppressor controls androgen signaling and human prostate cancer progression. J Clin Invest 2010;120:4478-92.

21. Altintas DM, Shukla MS, Goutte-Gattat D, Angelov D, Rouault JP, Dimitrov S, et al. Direct cooperation between androgen receptor and E2F1 reveals a common regulation mechanism for androgen-responsive genes in prostate cells. Mol Endocrinol 2012;26:1531-41.

22. Sharma A, Comstock CES, Knudsen ES, Cao KH, Hess-Wilson JK, Morey LM, et al. Retinoblastoma tumor suppressor status is a critical determinant of therapeutic response in prostate cancer cells. Cancer Re 2007;67:6192-203.

23. Bosco EE, Wang Y, Xu H, Zilfou JT, Knudsen KE, Aronow BJ, et al. The retinoblastoma tumor suppressor modifies the therapeutic response of breast cancer. J Clin Invest 2007;117:218-28

24. Thangavel C, Dean JL, Ertel A, Knudsen KE, Aldaz CM, Witkiewicz AK, et al. Therapeutically activating RB: reestablishing cell cycle control in endocrine therapy-resistant breast cancer. Endocr Relat Cancer 2011;18:333-45.

25. Heinz S, Benner C, Spann N, Bertolino E, Lin YC, Laslo P, et al. Simple combinations of lineage-determining transcription factors prime cis-regulatory elements required for macrophage and B cell identities. Mol Cell 2010;38: $576-89$.

26. Goodwin JF, Schiewer MJ, Dean JL, Schrecengost RS, de Leeuw R, Han S, et al. A hormone-DNA repair circuit governs the response to genotoxic insult. Cancer Discov 2013:3:1254-71.

27. Welti J, Sharp A, Yuan W, Dolling D, Rodrigues DN, Figueiredo I, et al. Targeting bromodomain and extra-terminal (BET) family proteins in castration-resistant prostate cancer (CRPC). Clin Cancer Res 2018;24:3149-62.

28. Polager S, Kalma Y, Berkovich E, Ginsberg D. E2Fs up-regulate expression of genes involved in DNA replication, DNA repair and mitosis. Oncogene 2002;21: $437-46$.

29. Hsieh MCF, Das D, Sambandam N, Zhang MQ, Nahlé Z. Regulation of the PDK4 isozyme by the Rb-E2F1 complex. J Biol Chem 2008;283:27410-7.

30. Blanchet E, Annicotte JS, Lagarrigue S, Aguilar V, Clapé C, Chavey C, et al. E2F transcription factor-1 regulates oxidative metabolism. Nat Cell Biol 2011;13 1146-54.

31. Augello MA, Hickey TE, Knudsen KE. FOXA1: master of steroid receptor function in cancer. EMBO J 2011;30:3885-94. 
32. Liu Y, Liu Y, Yuan B, Yin L, Peng Y, Yu X, et al. FOXM1 promotes the progression of prostate cancer by regulating PSA gene transcription. Oncotarget 2017;8 17027-37.

33. Kim MY, Jung AR, Kim GE, Yang J, Ha U-S, Hong S-H, et al. High FOXM1 expression is a prognostic marker for poor clinical outcomes in prostate cancer. J Cancer 2019;10:749-56.

34. Klokk TI, Kurys P, Elbi C, Nagaich AK, Hendarwanto A, Slagsvold T, et al. Ligand-specific dynamics of the androgen receptor at its response element in living cells. Mol Cell Biol 2007;27:1823-43.

35. Tan X, Martin SJ, Green DR, Wang JY. Degradation of retinoblastoma protein in tumor necrosis factor- and CD95-induced cell death. J Biol Chem 1997;272: 9613-6.

36. Chau BN, Borges HL, Chen T-T, Masselli A, Hunton IC, Wang JYJ. Signaldependent protection from apoptosis in mice expressing caspase-resistant $\mathrm{Rb}$. Nat Cell Biol 2002;4:757-65.

37. Han J, Soletti RC, Sadarangani A, Sridevi P, Ramirez ME, Eckmann L, et al. Nuclear expression of $\beta$-catenin promotes RB stability and resistance to TNFinduced apoptosis in colon cancer cells. Mol Cancer Res 2013;11:207-18.

38. Hilgendorf KI, Leshchiner ES, Nedelcu S, Maynard MA, Calo E, Ianari A, et al The retinoblastoma protein induces apoptosis directly at the mitochondria. Genes Dev 2013;27:1003-15.

39. You Z, Ouyang H, Lopatin D, Polver PJ, Wang CY. Nuclear factor-kappa Binducible death effector domain-containing protein suppresses tumor necrosis factor-mediated apoptosis by inhibiting caspase-8 activity. J Biol Chem 2001;276: 26398-404.

40. Kumar D, Whiteside TL, Kasid U. Identification of a novel tumor necrosis factoralpha-inducible gene, SCC-S2, containing the consensus sequence of a death effector domain of fas-associated death domain-like interleukin- 1betaconverting enzyme-inhibitory protein. J Biol Chem 2000;275:2973-8.

41. Niture S, Ramalinga M, Kedir H, Patacsil D. TNFAIP8 promotes prostate cancer cell survival by inducing autophagy. Oncotarget 2018;9:26884-99.

42. Zhang C, Kallakury BV, Ross JS, Mewani RR, Sheehan CE, Sakabe I, et al. The significance of TNFAIP8 in prostate cancer response to radiation and docetaxel and disease recurrence. Int J Cancer 2013;133:31-42.

43. Kaufmann SH, Desnoyers S, Ottaviano Y, Davidson NE, Poirier GG. Specific proteolytic cleavage of poly(ADP-ribose) polymerase: an early marker of chemotherapy-induced apoptosis. Cancer Res 1993;53:3976-85.

44. Salvesen GS, Riedl SJ. Caspase mechanisms. Adv Exp Med Biol 2008;615:13-23.

45. Kalin TV, Wang IC, Ackerson TJ, Major ML, Detrisac CJ, Kalinichenko VV, et al Increased levels of the FoxM1 transcription factor accelerate development and progression of prostate carcinomas in both TRAMP and LADY transgenic mice. Cancer Res 2006;66:1712-20.

46. Chandran UR, Ma C, Dhir R, Bisceglia M, Lyons-Weiler M, Liang W, et al. Gene expression profiles of prostate cancer reveal involvement of multiple molecular pathways in the metastatic process. BMC Cancer 2007;7:64.
47. Wang LL, Xiu YL, Chen X, Sun KX, Chen S, Wu DD, et al. The transcription factor FOXA1 induces epithelial ovarian cancer tumorigenesis and progression. Tumor Biol 2017;39:1010428317706210.

48. Yan Z, DeGregori J, Shohet R, Leone G, Stillman B, Nevins JR, et al. Cdc6 is regulated by E2F and is essential for DNA replication in mammalian cells. Proc Natl Acad Sci U S A 1998;95:3603-8.

49. Liu Y, Gong Z, Sun L, Li X. FOXM1 and androgen receptor co-regulate CDC6 gene transcription and DNA replication in prostate cancer cells. Biochim Biophys Acta 2014;1839:297-305.

50. Decker KF, Zheng D, He Y, Bowman T, Edwards JR, Jia L. Persistent androgen receptor-mediated transcription in castration-resistant prostate cancer under androgen-deprived conditions. Nucleic Acids Res 2012;40:10765-79.

51. Chen X, Müller GA, Quaas M, Fischer M, Han N, Stutchbury B, et al. The forkhead transcription factor FOXM1 controls cell cycle-dependent gene expression through an atypical chromatin binding mechanism. Mol Cell Biol 2013;33: 227-36.

52. Abida W, Cyrta J, Heller G, Prandi D, Armenia J, Coleman I, et al. Genomic correlates of clinical outcome in advanced prostate cancer. Proc Natl Acad Sci U S A 2019;166:11428-36.

53. Boussouar F, Jamshidikia M, Morozumi Y, Rousseaux S, Khochbin S. Malignant genome reprogramming by ATAD2. Biochim Biophys Acta 2013;1829:1010-4

54. Engelmann D, Pützer BM. The dark side of E2F1: in transit beyond apoptosis: Figure 1. Cancer Res 2012;72:571-5.

55. Zou JX, Guo L, Revenko AS, Tepper CG, Gemo AT, Kung H-J, et al. Androgeninduced coactivator ANCCA mediates specific androgen receptor signaling in prostate cancer. Cancer Res 2009;69:3339-46.

56. Liu W, Xie CC, Thomas CY, Kim S-T, Lindberg J, Egevad L, et al. Genetic markers associated with early cancer-specific mortality following prostatectomy. Cancer 2013;119:2405-12.

57. Byrne JA, Frost S, Chen Y, Bright RK. Tumor protein D52 (TPD52) and cancer-oncogene understudy or understudied oncogene? Tumor Biol 2014, $35: 7369-82$

58. Jagarlamudi KK, Shaw M. Thymidine kinase 1 as a tumor biomarker: technical advances offer new potential to an old biomarker. Biomark Med 2018;12:1035-48.

59. Kumar S, Talluri S, Pal J, Yuan X, Lu R, Nanjappa P, et al. Role of apurinic/ apyrimidinic nucleases in the regulation of homologous recombination in myeloma: mechanisms and translational significance. Blood Cancer J 2018;8:92.

60. Gökmen-Polar Y, Neelamraju Y, Goswami CP, Gu Y, Gu X, Nallamothu G, et al Splicing factor ESRP1 controls ER-positive breast cancer by altering metabolic pathways. EMBO Rep 2019;20:e46078.

61. Liu B, Li L, Yang G, Geng C, Luo Y, Wu W, et al. PARP inhibition suppresses GRMYCN-CDK5-RB1-E2F1 signaling and neuroendocrine differentiation in castration-resistant prostate cancer. Clin Cancer Res 2019;25:6839-51. 